\title{
Capacidad e integración fotovoltaica en edificios mixtos de mediana altura en la región ecuatorial andina
}

\author{
Paula Flores-Chafla ${ }^{1} \mid$ Daniel Pesantez-Peñafiel ${ }^{2} \mid$ Esteban Felipe Zalamea-León ${ }^{3} \mid$ Edgar Antonio \\ Barragán-Escandón ${ }^{4}$
}

Recibido: 11-05-2020 | en su versión final: 06-10-2020

Resumen Este trabajo proyecta cubiertas fotovoltaicas en un edificio de uso mixto de mediana altura, analizando el uso de tecnologías para diferentes niveles de integración arquitectónica, buscando máximo autoabastecimiento energético y reducción de emisiones. La metodología utilizada es la recolección y análisis de información desde lecturas de consumos temporales de medidores eléctricos con resolución cuarto-horaria, planillas de consumo eléctrico y registro, a través de encuestas, de la demanda de gas licuado de petróleo. Se analiza el consumo con datos acorde a la ocupación actual y se proyectan instalaciones fotovoltaicas considerando la ocupación teórica de distintas tecnologías. Con herramientas informáticas, se encuentra la adaptabilidad y ocupación fotovoltaica, y se simula la producción eléctrica, evidenciando las consecuencias de integración arquitectónica. Se determina que es posible incorporar hasta 338,4 m2 de captación con placas de silicio mono cristalino, pudiéndose alcanzar una producción de 196,8 kWh/m2/año y reduciendo potencialmente 47,2 t de CO2 anuales. Se estima un autoabastecimiento de hasta un 99,72\% con el nivel de consumo eléctrico actual. Pero, si se considera la conversión de combustibles usados en el condominio a electricidad, extrapolado a máxima ocupación de usuarios posible y la inclusión de cargadores para vehículos eléctricos, este potencial se reduciría a 27,85\%. Esto evidencia el importante potencial para introducir fuentes solares fotovoltaicas en el Ecuador dada la elevada y estable insolación anual promedio. Sin embargo, las políticas estatales y la falta de incentivos y esquemas de apoyo limitan la incorporación de estos sistemas.

Palabras clave: edificio multifamiliar; BAPV; BIPV; cubierta solar

Citación

\section{Photovoltaic Capacity and Integration in Mid-rise Multifamily Buildings in the Andean Equatorial Region}

Abstract

\begin{abstract}
This research analyzes photovoltaic roofs in a medium-size mixed-use building, evaluating solar technologies for different levels of architectural integration, with the goal of achieving maximum energy self-sufficiency and emission reductions. The implemented methodology collects and analyzes data from temporary electric consumption readings of electric meters with a quarter-hour resolution, electricity consumption sheets, and registration of the demand for liquefied petroleum gas through surveys. The electric consumption of the building is analyzed using current occupation data and the projection of photovoltaic installations is done considering the theoretical usage of different technologies. Computer tools are used to find the adaptability and photovoltaic occupation, and to simulate the electrical production, showing the consequences of architectural integration. The findings of the study show that it is possible to incorporate $338.4 \mathrm{~m}^{2}$ of mono crystalline silicon modules, reaching a production of $196.8 \mathrm{kWh} / \mathrm{m}^{2} /$ year and potentially reducing $47.2 \mathrm{t}$ of $\mathrm{CO}_{2}$ per year. It is estimated that energy self-sufficiency can reach up to $99.72 \%$ with current levels of electricity consumption. However, if the added electricity from the conversion of fuel consumption in the building to electricity is considered, extrapolated to the maximum possible user occupation and inclusion of electric chargers for vehicles, this estimate is reduced to $27.85 \%$. This demonstrates that there is a significant potential for introducing solar energy sources in Ecuador, given the high and stable average annual insolation of the country
\end{abstract} However, state policies and the lack of incentives and support limit the incorporation of these systems.

Keywords: multifamily building; BAPV; BIPV; solar roof

1 Arquitecta y técnico de investigación, Facultad de Arquitectura y Urbanismo, Universidad de Cuenca (ORCID: 0000-0003-2743-0855, WoS ResearcherID: AAF-9821-2021), ${ }^{2}$ Arquitecto, Facultad de Arquitectura y Urbanismo, Universidad de Cuenca (ORCID: 0000-0002-1085-7337, WoS ResearcherID: AAF-9455-2021), ${ }^{3}$ Dr. arquitecto. Profesor e investigador, Facultad de Arquitectura y Urbanismo, Universidad de Cuenca (ORCID: 0000-0001-5551-5026, WoS ResearcherID: H-7075-2016, Scopus Author ID: 57205768554), ${ }^{4}$ Dr. Ingeniero. Profesor e investigador, Departamento de Ingeniería Eléctrica, Universidad Politécnica Salesiana

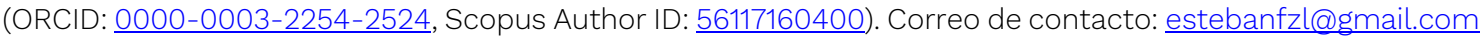




\section{Introducción}

El problema energético global motiva la búsqueda de medidas de eficiencia energética para lograr edificaciones y ciudades sostenibles reduciendo la afectación global por consumo de energía. Se requiere promover el autoabastecimiento energético en edificios, entre estos, aquellos de uso residencial-comercial, considerando se trata de una tipología que bajo la perspectiva de sostenibilidad tienen importantes ventajas adicionales, tanto desde el punto de vista social como energético (Hermida, María A; Hermida, Carla; Cabrera, Natasha; Calle, 2015).

Los países desarrollados tradicionalmente han sido los mayores emisores de gases de efecto invernadero; no obstante, la tendencia de crecimiento de estas emisiones es mayor en aquellos en vías de desarrollo. Pero problemas sociales urgentes implican que el asunto de emisiones sea relegado. Es necesario considerar que las sociedades en desarrollo son donde se prevé existirá más incremento de contaminación ambiental y también serán las más afectadas por los efectos del cambio climático. Concretamente en América Latina y el Caribe se prevé que la población crecerá a 762 millones de habitantes frente a los 653 millones existentes (United Nations, 2015). El crecimiento poblacional juntamente con un mayor desarrollo económico, tendrá como consecuencia un incremento de emisiones considerable. En Ecuador, la proyección poblacional para 2050 es de 23 ' 377 412 personas. Según datos del Ministerio de Electricidad y Energía Renovable el consumo energético nacional se incrementó 1.31 veces en 10 años (MEER, 2017). Se determina que en el año 2018 el 70.7\% de energía eléctrica utilizada provino de hidroeléctricas, y el 27.4\% de termoeléctricas; solo el 1.9\% se generó a partir de centrales de menor impacto ambiental como la eólica, solar, biogás y biomasa (ARCONEL, 2018a). A pesar del alto componente de hidroelectricidad en la matriz energética local, esta tecnología en explotación a gran escala actualmente es considerada como fuente no renovable debido al gran impacto causado al medio agreste como la deforestación, alteración de paisajes, reducción de caudales de río, entre otros (Lin \& Qi, 2017; Zhang et al., 2020). En el Ecuador es necesaria además la diversificación de la generación eléctrica, pues ello permitiría garantizar redes más fiables y menos sujetas a afectación por reducción de uno u otro recurso (Ponce-Jara et al., 2018).

Sin embargo, existen limitaciones para que estos recursos energéticos cumplan un papel importante frente a las energías no renovables, primordialmente por las políticas estatales, puesto que mediante los subsidios de costos al gas, gasolinas y electricidad, se incentiva el uso de fuentes contaminantes, resultando así las alternativas sustentables no rentables (Baquero L \& Quesada M., 2016). Además, es necesaria una reforma del marco legal, que considere la exoneración de impuestos y aranceles en la importación de estos equipos energéticos, e incentivos como precios preferenciales por la venta de electricidad a las empresas de distribución y facilidades para la conexión a la red eléctrica (Muñoz et al., 2018).

Para fomentar el uso de energías renovables, la Agencia de Regulación y Control de Electricidad (ARCONEL) con base en lo dispuesto en la Ley Orgánica del Servicio Público de Energía Eléctrica (LOSPEE), aprobó la regulación para la microgeneración fotovoltaica (PV) para autoabastecimiento de consumidores finales de energía eléctrica, en la cual se detallan los aspectos que deben cumplir los consumidores en cuanto a la implementación del sistema, su habilitación y el tratamiento comercial. Así, en referencia al mecanismo comercial, la empresa de distribución realizará el balance neto mensual de la energía entregada y consumida y, en caso de existir un remanente negativo; es decir, no lograr suplir la demanda, la empresa facturará la energía consumida, que no estará sujeta a ningún tipo de subsidio considerándose el costo real. Por el contrario, al generarse un eventual excedente de energía, este será considerado como un crédito energético a favor del consumidor para el mes siguiente, de manera consecutiva, hasta llegar al periodo máximo de 2 años en el que se realizará un reinicio. Además, es importante mencionar que de existir un crédito energético al llegar al periodo máximo o al dar de baja el suministro, el consumidor no recibirá ningún tipo de liquidación por el mismo (ARCONEL, 2018b). 
La autogeneración urbana es una alternativa que ayuda a disminuir la demanda en redes, donde la exigencia pico coincide con niveles de alta irradiación. La fuente solar resulta eficiente al abastecer en sitio, con ello se reduce pérdidas e impacto por redes de transmisión, y se evita afectar zonas agrestes por producción energética y transmisión (García, 2016).

Las edificaciones residenciales-comerciales son una tipología que, desde la perspectiva urbanística y de la sostenibilidad, resulta apropiada por la aptitud de minimizar problemas consecuentes como la especulación del suelo y reducción de espacio urbanizable, favoreciendo la consolidación en altura y evitando la dispersión urbana. Se promueve la densificación planificada resultando en una estrategia que coadyuva a la disminución de los impactos ambientales y socioeconómicos, evitando la ocupación del suelo rural. Promueve que habitantes residan próximos a servicios y fuentes de trabajo, y se fomenta la cercanía entre residencia y lugar de trabajo. Esta tipología también facilita la dotación de infraestructuras y servicios básicos, equipamientos comunitarios, transporte público, entre otros, indispensables para mejorar la calidad de vida (Hermida et al. 2015). Desde el punto de vista energético y del auto-aprovisionamiento solar, este tipo de configuración funcional diversa tiene como resultado que las curvas de consumo energético residencial, frente a servicio o comercio, tienden a equilibrar los desbalances horarios típicos. La carga energética residencial es mayormente pronunciada temprano en la mañana y al final de la tarde, contrario a los usos de servicio y comercio en los que el consumo es mayor en horas cercanas al medio día (Hachem, 2015). Sin embargo, la densificación implica a la vez una menor incidencia de irradiación para aprovechamiento pasivo y activo, siendo necesario maximizar la utilización del recurso energético a través del diseño.

Para aprovechamiento solar en arquitectura como complemento a la captación pasiva, dos tecnologías activas son típicamente aplicadas: los colectores solares térmicos (CSTs) y fotovoltaicos (PVs), los cuales pueden encontrarse en las edificaciones colocados o integrados arquitectónicamente en las cubiertas (Shukla et al., 2016). Los CSTs son tecnológicamente más sencillos, prácticos y eficientes para abastecer calor; no obstante, los PVs han mostrado un acelerado desarrollo y eficiencia, y su masificación ha implicado una reducción de costos dramática y un crecimiento sustancial (Zalamea \& Quesada, 2017). Este trabajo analiza el potencial de los colectores PVs dada la versatilidad de la electricidad para resolver todo tipo de demandas residenciales. En la Tabla 1 se expone un análisis de edificios de similar configuración de altura media, uso residencial y mixtos, con integración de tecnologías solares para energización, la respectiva producción anual, el porcentaje de autoabastecimiento y la reducción de emisiones de $\mathrm{CO}_{2}$.

Tabla 1. Casos de estudio de edificios multifamiliares con tecnologías de captación solar

\begin{tabular}{|c|c|c|c|c|c|c|c|}
\hline Proyecto & País & $\begin{array}{c}\# \\
\text { dptos. }\end{array}$ & Ubicación & $\begin{array}{l}\text { Demanda } \\
\text { anual }\end{array}$ & $\begin{array}{l}\text { Producción } \\
\text { anual }\end{array}$ & $\begin{array}{l}\% \text { Abasteci- } \\
\text { miento }\end{array}$ & $\mathrm{CO}_{2}$ \\
\hline Kraftwerk B & Suiza & 7 & $\begin{array}{c}\text { PVs cubierta } \\
261 \mathrm{~m}^{2} / \\
\text { CSTs Fachada } \\
146 \mathrm{~m}^{2}\end{array}$ & $62 \mathrm{MWh}$ & $\begin{array}{c}32 \mathrm{MWh} \text { PV } \\
38 \mathrm{MWh} \text { CST }\end{array}$ & $113 \%$ & $48 t$ \\
\hline Järvenpää & Finlandia & 44 & $\begin{array}{l}\text { CSTs cubierta } \\
126 \mathrm{~m}^{2} \text { y PVs } \\
\text { aleros } 108 \mathrm{~m}^{2}\end{array}$ & $217 \mathrm{MWh}$ & $196 \mathrm{MWh}$ & $90 \%$ & $76 t$ \\
\hline $\begin{array}{c}\text { Dieselweg } \\
\text { Graz }\end{array}$ & Austria & 204 & $\begin{array}{l}\text { CSTs cubierta } \\
126 \mathrm{~m}^{2} \text { y PVs } \\
\text { aleros } 108 \mathrm{~m}^{2}\end{array}$ & --- & --- & $90 \%$ & $89 \%$ \\
\hline $\begin{array}{c}\text { Alessandria } \\
\text { Photovoltaic } \\
\text { Village }\end{array}$ & Italia & 304 & $\begin{array}{c}\text { PVs cubierta y } \\
\text { fachada }\end{array}$ & --- & $160 \mathrm{MWh}$ & $\begin{array}{l}\text { 100\% electr. } \\
\text { áreas } \\
\text { comunes y } \\
70 \% \text { dptos.. }\end{array}$ & $100 t$ \\
\hline
\end{tabular}

ACE, 15 (45) CC BY-ND 3.0 ES | UPC Barcelona, España | Capacidad e integración fotovoltaica en edificios 3 multifamiliares de mediana altura en la región ecuatorial andina. DOI: http://dx.doi.org/10.5821/ace.15.45.9307 


\section{ACE Architecture, City and Environment}

E-ISSN $1886-480.5$

\begin{tabular}{|c|c|c|c|c|c|c|c|}
\hline Proyecto & País & $\begin{array}{c}\# \\
\text { dptos. }\end{array}$ & Ubicación & $\begin{array}{l}\text { Demanda } \\
\text { anual }\end{array}$ & $\begin{array}{l}\text { Producción } \\
\text { anual }\end{array}$ & $\begin{array}{c}\% \text { Abasteci- } \\
\text { miento }\end{array}$ & $\mathrm{CO}_{2}$ \\
\hline Kleehäuser & Alemania & 25 & $\begin{array}{l}\text { PVs } 202 \mathrm{~m}^{2} \mathrm{y} \\
\text { CSTs } 61.2 \mathrm{~m}^{2} \\
\text { cubierta }\end{array}$ & $40 \mathrm{MWh}$ & $\begin{array}{c}23 \mathrm{MWh} \text { PV } \\
17 \mathrm{MWh} \text { CST }\end{array}$ & $100 \%$ & $16 t$ \\
\hline $\begin{array}{c}\text { Edificio } 1 \\
(1909)\end{array}$ & Suiza & 32 & $\begin{array}{l}\text { PVs cubierta y } \\
\text { balaustrada }\end{array}$ & $\begin{array}{l}\text { 1.2 } \mathrm{MWh} / \mathrm{m}^{2} \\
\text { caldera } \\
0.8 \mathrm{MWh} / \mathrm{m}^{2} \\
\text { bomba calor }\end{array}$ & $\begin{array}{l}14 \mathrm{MWh} \\
\text { caldera; } \\
28 \mathrm{MWh} \\
\text { bomba calor }\end{array}$ & $\begin{array}{c}24.5 \% \text { caldera } \\
29.0 \% \text { bomba } \\
\text { calor }\end{array}$ & $30 \%$ \\
\hline $\begin{array}{c}\text { Edificio } 2 \\
(1972)\end{array}$ & Suiza & 52 & $\begin{array}{l}\text { PVs fachada y } \\
\text { barandilla }\end{array}$ & $\begin{array}{c}0.8 \mathrm{MWh} / \mathrm{m}^{2} \\
\text { caldera } \\
0.6 \mathrm{MWh} / \mathrm{m}^{2} \\
\text { bomba calor }\end{array}$ & $\begin{array}{c}87 \mathrm{MWh} \\
\text { caldera } \\
139 \mathrm{MWh} \\
\text { bomba calor }\end{array}$ & $\begin{array}{c}29.0 \% \text { caldera } \\
32.0 \% \text { bomba } \\
\text { calor }\end{array}$ & $30 \%$ \\
\hline
\end{tabular}

Fuente: Elaboración propia con base en la información de Aguacil, Lufkin, \& Rey, 2017; Baur-Gschier, Stückler, \& Mayrhofer, 2014; Erhorn \& Erhorn-Kluttig, 2014; Gaiddon, 2009; Voss \& Musall, 2012.

Los casos referenciados son en países con clima estacional marcado. No se ha podido detectar estudios similares bajo las características climáticas ecuatoriales, y menos aún andinas (en valles altos). El presente estudio se desarrolla en la ciudad de Cuenca, Ecuador (2535 msnm) lo que conlleva condiciones peculiares. Es un clima templado con variación estacional mínima, con máximas de 25C y mínimas de 8C durante todo el año (Climate-Data.org, 2014). Una particularidad es el contexto en general: tanto unidades habitacionales como comerciales no requieren sistemas de ambientación gracias al favorable clima, lo cual es una situación que supone una gran aptitud para potencializar la captación solar y por ende alcanzar máximos niveles de autoaprovisionamiento (Figura 1). Bajo estas condiciones, la alternativa para lograr reducciones de consumo energético urbano y de las edificaciones, es integrar energías renovables, dado que no existen exigencias ambientales que requieran maximizar estrategias pasivas. Adicional al análisis energético, este estudio observa distintos grados de integración arquitectónica, considerando simultáneamente capacidad energética y de afectación visual-urbanística. Finalmente, se mide el potencial de autoaprovisionamiento del edificio al convertir a electricidad todas las demandas cubiertas, por ahora, con combustibles y se analiza una posible alimentación a vehículos eléctricos (EVs).

Figura 1. Contexto de emplazamiento, edificio Pinar del Lago I

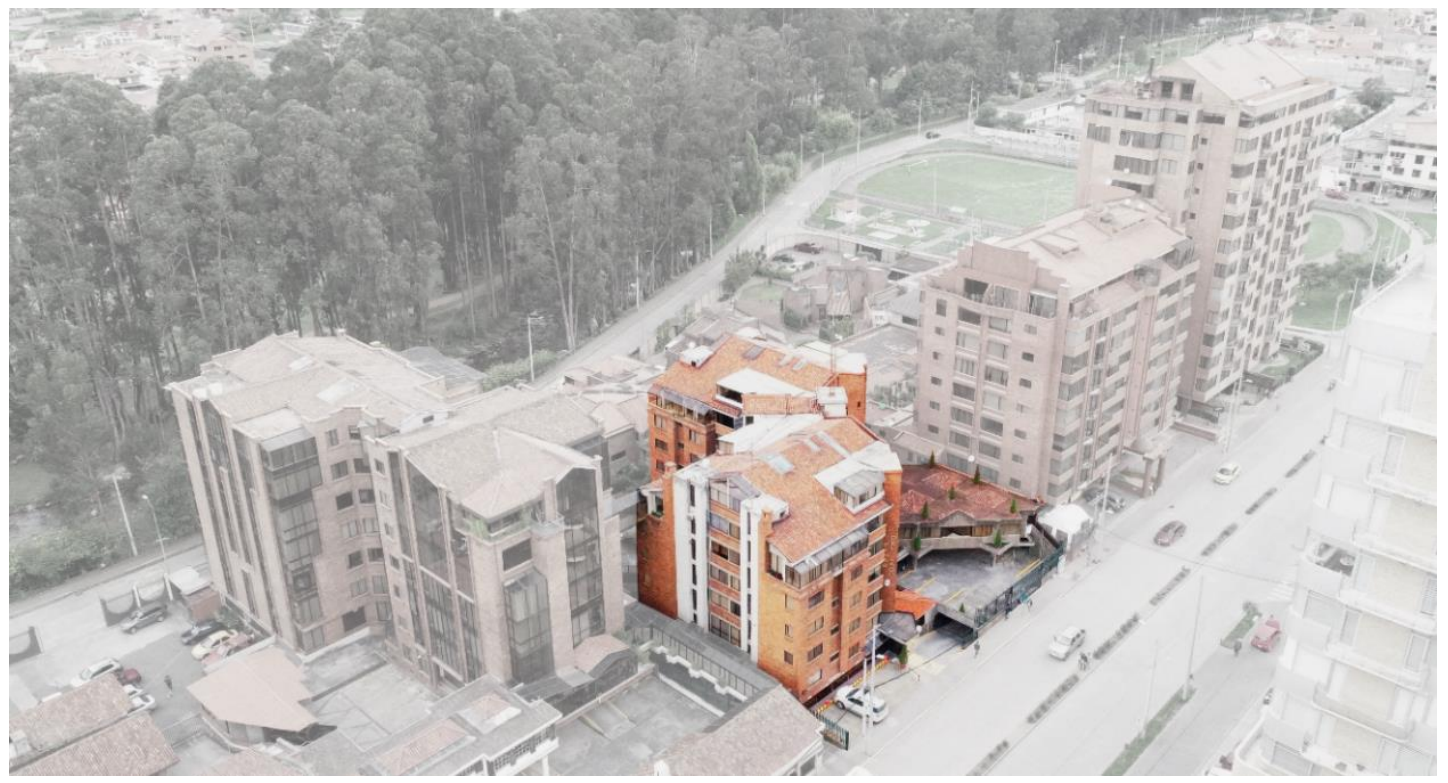

Fuente: Elaboración propia.

ACE, 15 (4.5) CC BY-ND 3.0 ES | UPC Barcelona, España | Capacidad e integración fotovoltaica en edificios multifamiliares de mediana altura en la región ecuatorial andina. DOI: http://dx.doi.org/10.5821/ace.15.45.9307 
El caso analizado comprende una construcción en 3 bloques, 2 de siete plantas de uso residencialcomercial y 1 de dos pisos destinado a oficinas. En los dos bloques principales funcionan: en la planta baja 2 departamentos con una habitación y en la planta baja más las dos últimas funcionan oficinas; entre la segunda y cuarta plantas altas se emplazan 15 departamentos para 4 personas y 4 departamentos para 2 personas. La edificación está estructurada en hormigón con mampostería de ladrillo y planos de vidrio. La cubierta es de teja, vidrio y losas de hormigón armado. La techumbre se desarrolla a dos aguas con una pendiente del 35\%, inclusive en la parte central de ambos bloques. Solo dos faldones son de superficie menor, y presentan inclinación del 25\%. Para captar la energía solar la condición y techumbre disponible es fundamental. Existe una superficie total de $654.64 \mathrm{~m}^{2}$ distribuidos de la siguiente manera (Figura 2): $390.45 \mathrm{~m}^{2}$ faldones de 1 al 6 de teja artesanal y P=35\%, $5.04 \mathrm{~m}^{2}$ faldones 7 y 8 de teja artesanal $\mathrm{P}=25 \%, 156.59 \mathrm{~m}^{2}$ de losa plana de hormigón armado y 99.56 $\mathrm{m}^{2}$ corresponden a vidrio.

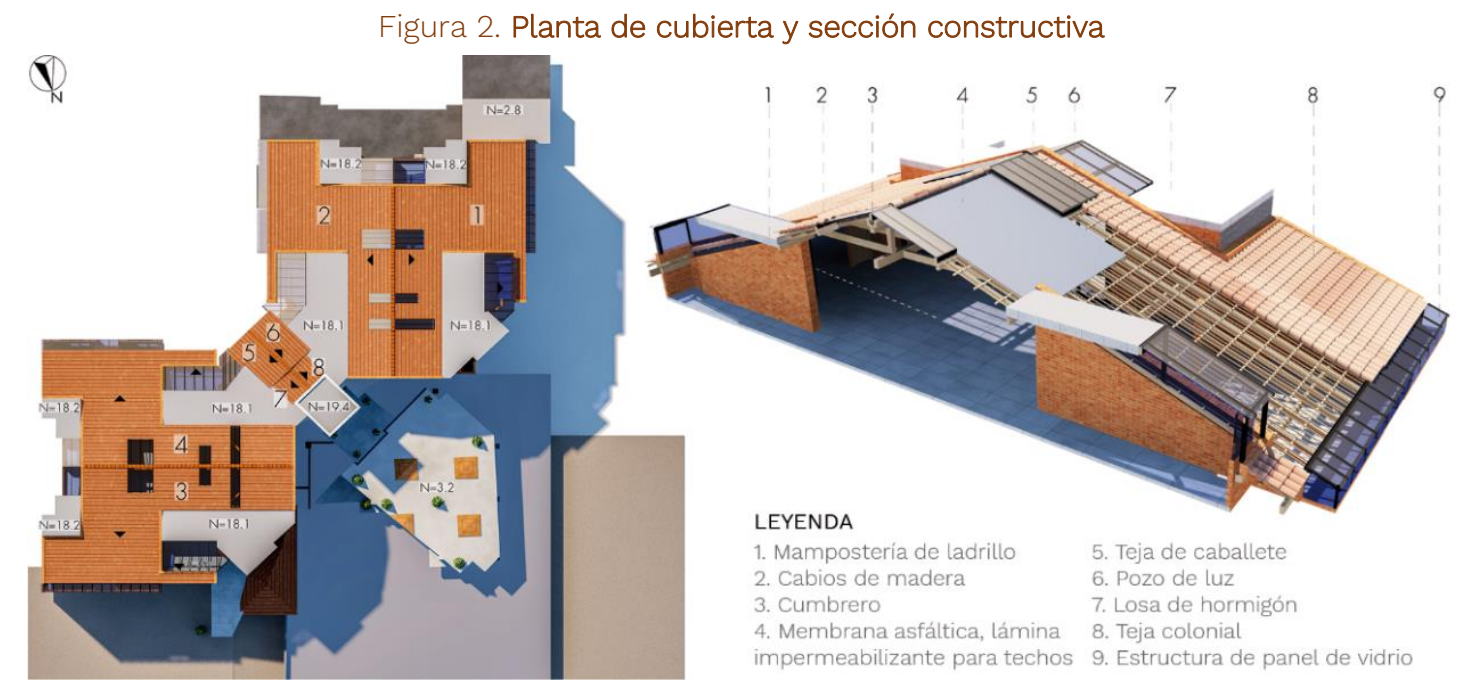

Fuente: Elaboración propia.

\section{Metodología}

Se aplican técnicas de recopilación y procesamiento de datos cuantitativos, permitiendo que el estudio sea descriptivo-concluyente. Se empleó análisis estadístico de la información primaria obtenida del consumo de energía eléctrica y Gas Licuado de Petróleo (GLP), así como de datos reales de consumo proporcionados por la empresa eléctrica local y mediciones en sitio con dispositivos para registro temporal de consumo A1800 ALPHA Meter. Las simulaciones de rendimiento PV se realizaron a través del software SAM, mientras que en ArchiCad de Graphisoft y Ecotec de Autodesk, se modeló la geometría y visualizaron indicadores de sombras. Finalmente, se realizó un análisis de resultado arquitectónico a través de fotomontajes. Con ello, el estudio cumplió las siguientes fases:

- Análisis del nivel de ocupación actual y máximo para lo que se diseñó la edificación, con la recolección y caracterización de información de consumo por unidad residencial y comercial.

- Identificación de consumos energéticos, a partir de información estadística anual disponible en la página web de la Empresa Eléctrica Regional Centro Sur y fluctuación del consumo eléctrico del edificio, a través de toma de datos con dispositivos para registro temporal de consumo A1800 ALPHA Meter instalados en los medidores. Registro de consumo de GLP desde encuestas, determinando el destino de consumo.

- Estimación de capacidad de abastecimiento PV a partir de modelo de simulación SAM y archivo climático de un año (Izquierdo et al., 2019). 
- Propuesta de integración arquitectónica aplicando captación solar con tecnologías PVs y extrapolación a consumos previstos considerando máxima ocupación, conversión de usos de combustibles a electricidad e inclusión de un cargador para EV por departamento.

\subsection{Diseño de investigación}

El estudio fue experimental, ya que se manipularon las variables de análisis deliberadamente, analizando el comportamiento actual de consumos de energía y proyectando demandas a electricidad. De igual manera, se manejaron las variables de generación de energía mediante la simulación de implementación PV. Al tomar en cuenta los consumos de enero a diciembre en los años 2018 y 2019 comparativamente, se adoptó el más crítico. Con los datos identificados de consumos promedio diario, mensual y anual se seleccionó productos PVs con distinto grado de integración arquitectónica. Se estableció el rediseño de la cubierta partiendo de tecnologías para adhesión coplanar arquitectónica Building Added Photovoltaics (BAPV) y tecnología para mimetización arquitectónica Building Integrated Photovoltaics (BIPV), considerando datos como la superficie, pendiente, inclinación y ángulo azimut de cada faldón ya existente, los cuales permitieron identificar los tipos de PVs, dimensiones, rendimientos, potencia y niveles de voltaje con los que operan los productos.

Para la propuesta constructiva, se toma como referencia todas las características técnicas de los paneles y las condiciones de montaje para los tres tipos de tecnologías: a) Panel de silicio monocristalino de 60 celdas, b) Panel de silicio monocristalino PERC (Passivated Emitter and Rear Cell) sin contactos metálicos entre células; y c) Tejas PVs de silicio monocristalino. El análisis es referencial a modo de estimación de prefactibilidad en el caso de las placas convencionales, ya que el modelo de simulación ha sido calibrado con precisión. En los casos del producto sin contacto metálico y de las tejas PVs, la eficiencia fue obtenida a partir de la literatura consultada, pues el rendimiento del producto no ha sido validado localmente. Las implicaciones arquitectónicas que tendría la integración de las tecnologías a la cubierta para cada alternativa se clasifican según niveles técnicos y formales. Adicionalmente, se estima el costo aproximado de inversión y el respectivo tiempo de amortización durante la vida útil de las tecnologías mediante la comparación de dos escenarios: con el precio oficial de energía eléctrica subsidiado según la Estadística Anual y Multianual del Sector Eléctrico Ecuatoriano 2018 (ARCONEL, 2018a), y considerando el costo real de generación y distribución sin subsidio.

Finalmente, se pone a consideración un análisis comparativo contemplando los siguientes escenarios: a) Rendimiento energético, b) Integración arquitectónica y c) Accesibilidad económica. Este trabajo presenta la novedad de ejecutar estos análisis en conjunto de estos aspectos, además de desarrollarse en localización ecuatorial, entregando un aporte significativo para el desarrollo de la electrificación en regiones similares, siendo el primero en desarrollarse bajo estas variables, hasta donde se pudo conocer. Complementariamente, se entregan los resultados a la empresa local de distribución para su consideración en el escenario de conexión a red.

\section{Caracterización de consumos y demandas energéticas del edificio}

De los 19 departamentos existentes, dos se encuentran deshabitados y seis presentan una ocupación intermitente a lo largo del año. A la fecha de la toma de datos el condominio está al 48.6\% de su posibilidad máxima de ocupación, considerando el número de habitaciones por unidad. No obstante, la ocupación en oficinas es de alrededor de cien personas, pero con ocupación intermitente. Se toma este como caso base y se extrapola a la máxima demanda previsible más la conversión a electricidad de los actuales consumos de combustible, incluyendo vehículos. Cabe mencionar que se recopiló el

ACE, 15 (45) CC BY-ND 3.0 ES | UPC Barcelona, España | Capacidad e integración fotovoltaica en edificios 6 multifamiliares de mediana altura en la región ecuatorial andina. DOI: http://dx.doi.org/10.5821/ace.15.45.9307 
consumo real emitido directamente de la empresa distribuidora de energía eléctrica de la ciudad, ya que son datos cuantitativos precisos y se prioriza observar el desempeño frente a una realidad.

\subsection{Consumo eléctrico}

Al instalar el equipo de monitoreo en 15 medidores con demanda residencial, 4 comerciales y 1 comunitario, se midieron las fluctuaciones horarias de consumo. También se contabilizó que los consumos registrados en los años 2018 y 2019 fueron de 46503.73 kWh y 48468.06 kWh, con un costo de $\$ 4459.87$ y $\$ 4683.74$, respectivamente, a precio subsidiado, considerando un costo real de electricidad de $\$ 0.16$ frente a costos de venta de $\$ 0.09$.

La Figura 3 marca las curvas del consumo mensual residencial al 2018 en las que se evidencia que no existen variaciones marcadas a lo largo del año. El mes de mayor consumo fue diciembre (2595.90 kWh) y el de menor enero (1580.67 kWh); con un promedio de 1956.38 kWh. En tanto que, en el 2019, se evidenciaron fluctuaciones con un comportamiento contrario hasta julio (mes de menor consumo 1792.40 kWh), elevándose hasta diciembre (3448.54 kWh). El consumo mensual promedio de 2019 fue de 2164.46 kWh, un 9.6\% superior respecto al anterior. Se recalca que son mediciones reales facturadas por la empresa de distribución.

Figura 3. Consumo eléctrico anual del edificio

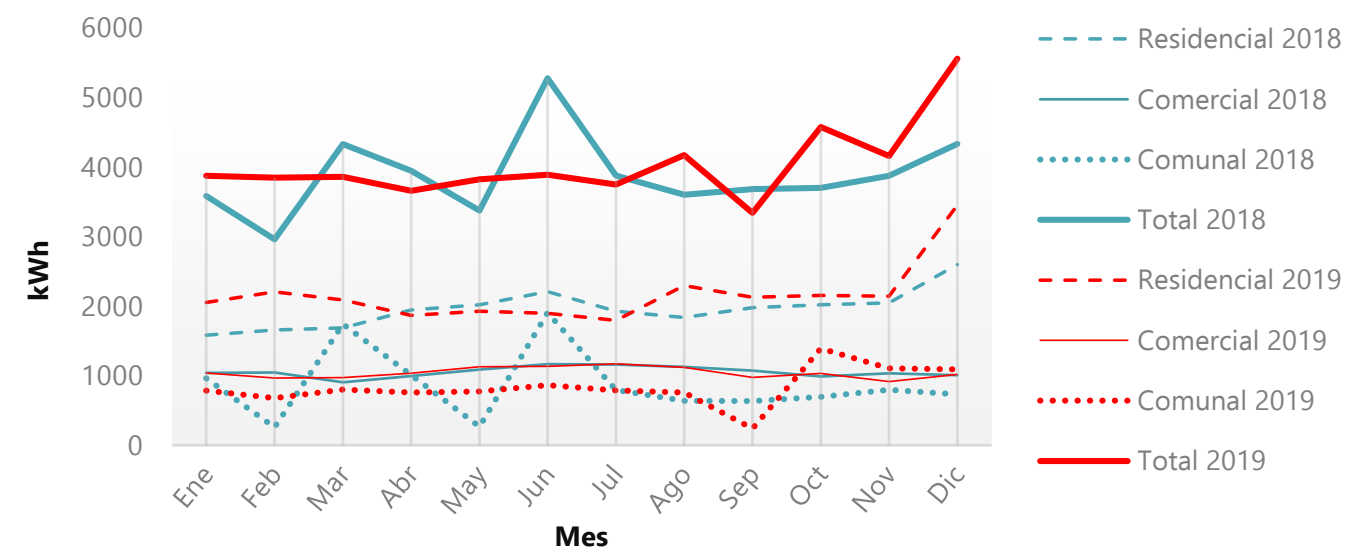

Fuente: Elaboración propia con base en la información obtenida de la Empresa Eléctrica Regional Centro Sur.

En el consumo comercial se evidencian curvas con menor variación, presentando un promedio mensual entre 1050.94 kWh y 1040.27 kWh en 2018 y 2019, respectivamente. En ambos casos el mes con mayor consumo fue julio con 1166.88 kWh; contrariamente, los meses más bajos son marzo (2018) y noviembre (2019). Por otro lado, el consumo comunitario de 2018 muestra oscilaciones marcadas de enero a julio y un aumento constante hasta diciembre. El mes de mayor consumo identificado fue junio (1904.34 kWh) y el de menor febrero (257.04 kWh); es decir, siete veces más. La variación de consumo no evidencia una clara relación con períodos climáticos del año, más bien la tendencia es un leve incremento a fin de año, asociado a tratarse de una época de mayor comercio y festivo. El consumo promedio mensual fue de $868.00 \mathrm{kWh}$ (Figura 3).

En cuanto a oscilación de consumo diario, el promedio alcanzó un valor de 120.52 kWh siendo el valor mínimo de 88.16 kWh (sábado) y máximo de 138.07 kWh (martes); datos que fueron registrados durante 3 meses a través de contadores de energía con medición cuarto-horaria, colocados en cada departamento. Los consumos diarios de energía del condominio son marcadamente distintos entre días laborables y fines de semana; cuya hora pico está entre las 09:00 y 10:00 h, seguido por un

ACE, 15 (45) CC BY-ND 3.0 ES | UPC Barcelona, España | Capacidad e integración fotovoltaica en edificios 7 multifamiliares de mediana altura en la región ecuatorial andina. DOI: http://dx.doi.org/10.5821/ace.15.45.9307 
segundo pico de consumo entre las 16:00 y 18:00 horas (Figura 4a). En la Figura 4b se observa desglosado entre consumo residencial y comercial.

Figura 4. Consumo eléctrico horario del edificio

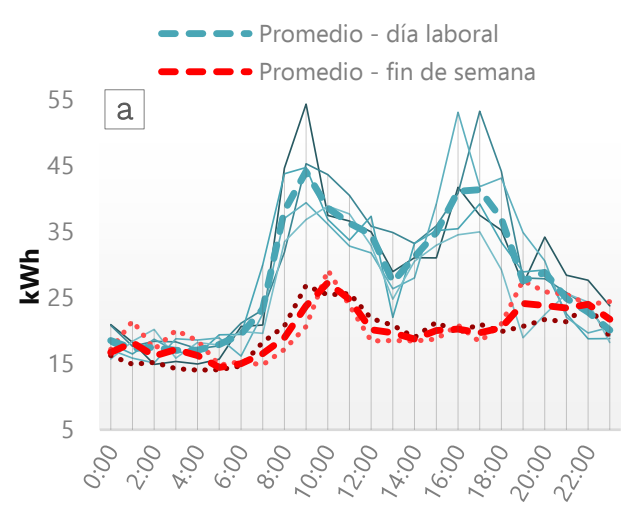

Hora

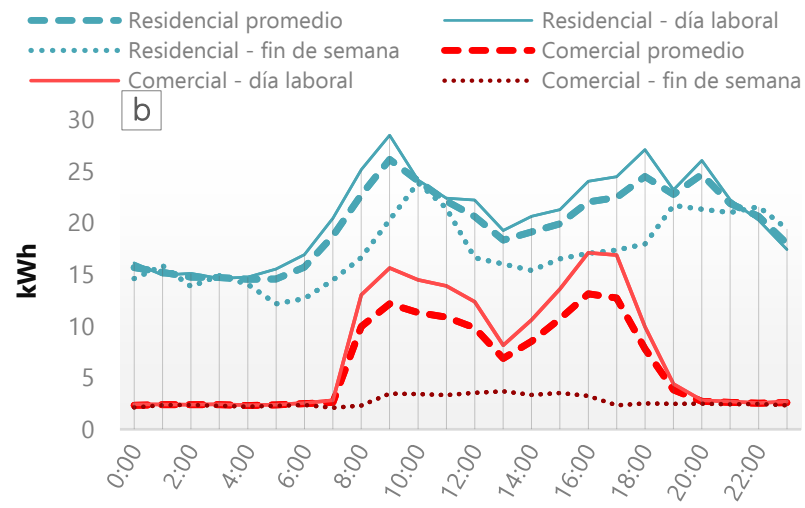

Hora

Fuente: Elaboración propia en base a la información registrada por los medidores cuarto-horarios.

El promedio mensual por unidad habitacional registrado en 2019 fue de $137.68 \mathrm{kWh}$, variable en un rango entre $57.65 \mathrm{kWh}$ y $177.06 \mathrm{kWh}$, dependiente del número de habitantes. Se estimó entonces, que el consumo eléctrico mensual por habitante residencial en este tipo de edificación fue de aproximadamente $45.1 \mathrm{kWh}$.

\subsection{Consumo térmico}

En Ecuador, el GLP es un combustible subsidiado para uso residencial, utilizado significativamente para la cocción y agua caliente sanitaria (ACS). En el condominio se contabilizó el uso de 18 cilindros de $15 \mathrm{~kg}$ mensualmente para el total del edificio, con una mediana de 2 cilindros por departamento, destinando el 50\% a la cocción de alimentos, 40\% para calentamiento de agua y el 10\% restante para secado y reserva. Los locales de oficina no registran consumo de combustibles.

El consumo de gas respecto al eléctrico es comparable acorde al uso y eficiencia del equipo equivalente eléctrico, así la relación de un cilindro de $15 \mathrm{~kg}$ de GLP posee una capacidad energética de 200.62 kWh (Tama, 2013). Pero la eficiencia energética es distinta, es así que una cocina de combustión a gas tiene 40\%, la de inducción eléctrica 84\% (Tama, 2013), el calefón a gas alrededor del 97\% y la bomba de calor para calentamiento de agua un 350\% (Villalta \& Buñay, 2016). Realizando la conversión al consumo de los departamentos desde GLP a kWh en equipos eléctricos, los requerimientos mensuales serían: a) 722.2 kWh en cocinas de inducción; b) 402.2 kWh considerando el uso de bombas de calor para ACS y c) $401.2 \mathrm{kWh}$ para reserva de propietario (no determinado) y secadoras de ropa. Con ello, se requieren $1525.63 \mathrm{kWh}$ mensualmente.

Por otro lado, el costo estimado con el subsidio estatal al GLP de $15 \mathrm{~kg}$ es de \$1.60, equivalente a $\$ 28.80$ en todo el condominio. Ello supone un costo real de \$15 (valor de venta del GLP no residencial sin subsidio) que estaría en torno a los $\$ 270$ mensual de gasto en precio real.

\subsection{Consumo total del edificio}

Realizando la conversión de consumo de combustible a electricidad en período mensual, y considerando conjuntamente las demandas residencial, comercial y comunitario, se determinó que

ACE, 15 (4.5) CC BY-ND 3.0 ES | UPC Barcelona, España | Capacidad e integración fotovoltaica en edificios 8 multifamiliares de mediana altura en la región ecuatorial andina. DOI: http://dx.doi.org/10.5821/ace.15.45.9307 
el edificio con la ocupación habitacional actual requiere aproximadamente: $4039.01 \mathrm{kWh}$ en electricidad y 1525.63 kWh en combustibles; es decir, un total de 66775.62 kWh al año. En términos económicos, esto representa un saldo de \$556.46 al mes en promedio. En caso de aprovechar al máximo las condiciones habitacionales del edificio, se necesitarían 4309.23 kWh al mes para suplir la demanda eléctrica, 7691.77 kWh mensuales para remplazar el uso de GLP. Esto se estimó empleando el consumo residencial habitacional de 2018, que fue de 135.26 kWh (ARCONEL, 2018a) y la estadística nacional de cilindros de GLP consumidos mensualmente de 2.13, para familia de 4 personas (Vicente Ramón, 2017). Si se incorpora la alimentación de EV de los habitantes del condominio, (3396.9 kWh al mes por auto, considerando un recorrido diario de $20 \mathrm{~km}$ promedio), para $11 \mathrm{EV}$ se necesitarían $102177.18 \mathrm{kWh}$ al año.

\section{Estimación de irradiación disponible en techumbre}

Se analiza la incidencia solar en la techumbre y sombras a través del software Ecotec Analysis, a partir del modelo 3D de la totalidad del edificio y de los bloques adyacentes. El tiempo establecido para la simulación partió desde las 09:00 hasta las 17:00 horas, en los solsticios de invierno y verano (21 de junio y 21 de diciembre) para determinar los desplazamientos de sombra máximos.

Se observó que la incidencia solar en el solsticio de invierno (21 de junio) es la menos favorable del año, ya que 2 faldones se encuentran parcialmente sombreados, uno de ellos hasta en un $74 \%$ de su superficie. Únicamente dos faldones conservaron el 100\% de iluminación durante todo el día (Figura 5). No obstante, la proyección de sombras empieza a afectar a partir de las 15:00 h, cuando el nivel de irradiación empieza a reducirse, por lo que la captación se ve mínimamente afectada.

Figura 5. Representación de sombras - solsticio de invierno 21 de junio

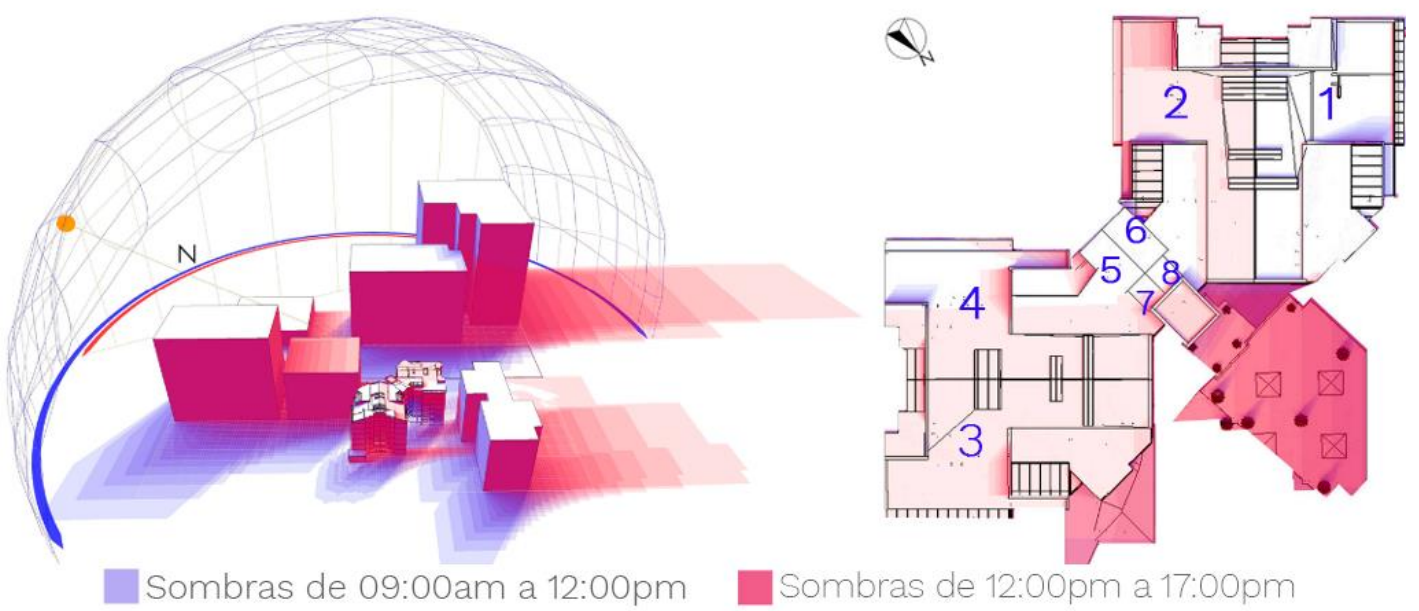

Fuente: Elaboración propia.

En tanto que, en el solsticio de verano (21 de diciembre), la incidencia de los niveles de sombra es menos significativa y solo un $24 \%$ de la totalidad de la cubierta está en sombra a las 17:00 h. En la Figura 6 se la puede observar prácticamente iluminada en su totalidad, con una afectación menor que empieza a partir de las 15:00 h. Se evidencia que todos los faldones son aptos para generación PV con afectación mínima por bloqueo solar. A pesar de que la edificación es baja respecto a los edificios inmediatos, casi no percibe sombra por el desplazamiento solar ecuatorial.

ACE, 15 (45) CC BY-ND 3.0 ES | UPC Barcelona, España | Capacidad e integración fotovoltaica en edificios 9 multifamiliares de mediana altura en la región ecuatorial andina. DOI: http://dx.doi.org/10.5821/ace.15.45.9307 
Figura 6. Representación de sombras - solsticio de verano 21 de diciembre
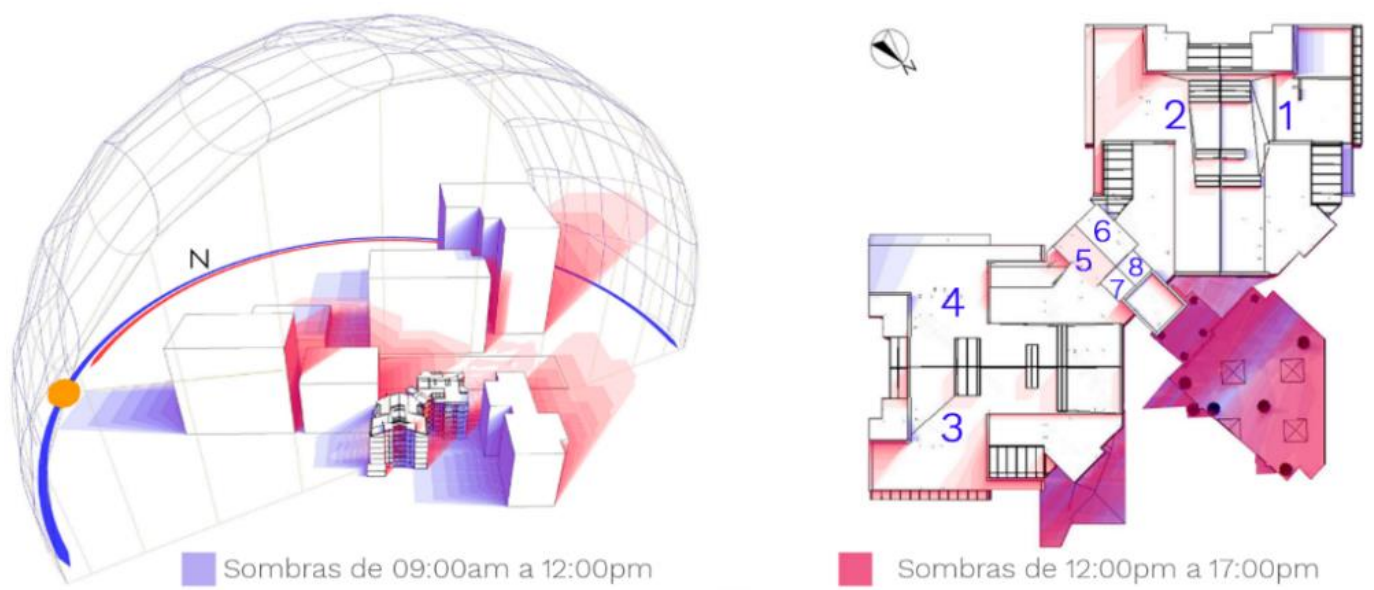

Fuente: Elaboración propia.

\section{Propuesta de rediseño de cubierta}

Se determinan aspectos generales para las tres tecnologías PVs descritas en la metodología respecto a adaptabilidades geométricas de faldones, pendiente, inclinación y azimut, que son detalles que inciden en colocación de PVs y, por ende, su rendimiento. Se plantean modificaciones menores en la techumbre para desplegar o integrar los PVs, respetando la iluminación cenital existente, aunque con modificaciones leves en concordancia con la geometría de los PVs.

A continuación, se detallan las diferentes tecnologías aplicadas a la investigación basándose en un mercado internacional, específicamente en los Estados Unidos de América debido a su facilidad de comercialización y aplicación en cuanto a tecnología PV. Cabe mencionar que se tomó en cuenta la certificación Tier 1 para paneles solares, misma que se usa en la industria solar como un ranking para fabricantes PVs, de acuerdo a su nivel de eficacia, solvencia y estabilidad económica. Dentro de esta categoría solo se registran fabricantes aceptados por la empresa Bloomberg New Energy Finance Corporation de América del Norte (Solar Review, 2020).

\subsection{Panel de silicio monocristalino de 60 celdas (BAPV)}

Son placas PVs típicas monocristalinas, productos ampliamente difundidos en el mercado local. Estas placas son las más comunes para el uso residencial y en edificaciones, por ser económicas y generalizadas. Se determinó un rendimiento de eficiencia promedio de $15.50 \%$ en el contexto de estudio (Izquierdo et al., 2019). El panel PV Q.ANTUM 285W Solar Panel Q.PLUS, proveniente de los fabricantes QCELLS, tiene unas dimensiones de 1670×1000×32 mm, entrega un total de 285 W / 31.99 $\checkmark$, y posee un marco de aluminio de alta calidad de $32 \mathrm{~mm}$ (QCELLS, 2015).

En este caso se desplegó sobre la techumbre un total de 208 unidades de paneles PVs de 60 celdas. La fijación a la cubierta se da por medio de perfiles que se acoplan sobre la cubierta existente y se anclan los paneles PVs sobre los perfiles mencionados, conservando la techumbre actual de la edificación. Se consideran productos de adaptación arquitectónica ya que solo cumplen con la función de generación eléctrica y no como cobertura de techumbre (Kaan \& Reijenga, 2004) (Figura 7).

ACE, 15 (45) CC BY-ND 3.0 ES | UPC Barcelona, España | Capacidad e integración fotovoltaica en edificios 10 multifamiliares de mediana altura en la región ecuatorial andina. DOI: http://dx.doi.org/10.5821/ace.15.45.9307 


\section{ACE Architecture, City and Environment}

E-ISSN $1886-4805$

Figura 7. Perspectiva con paneles de silicio monocristalino y detalle constructivo

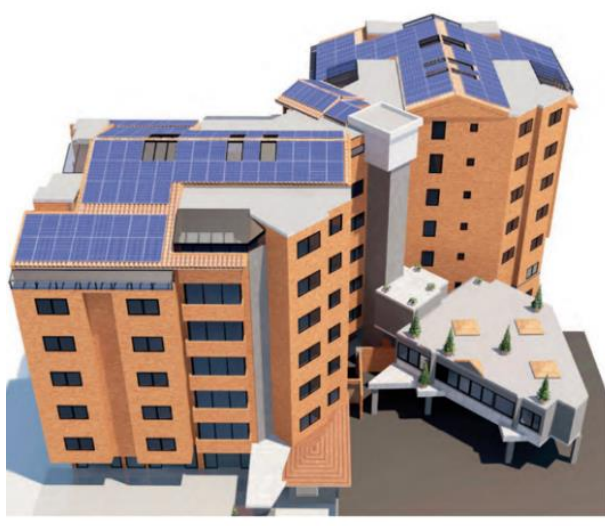

Fuente: Elaboración propia.

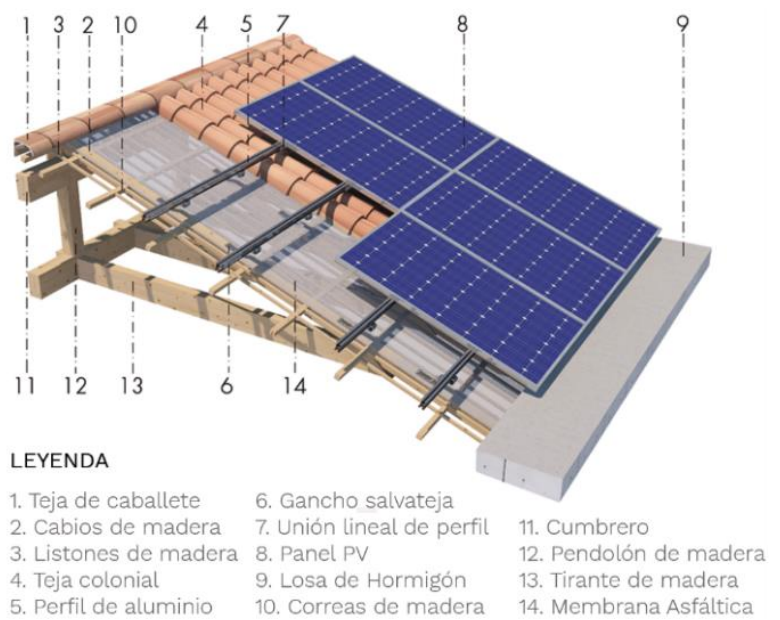

\subsection{Panel PV de silicio monocristalino PERC negro sin contactos metálicos entre células (BAPV)}

Estos productos poseen un alto rendimiento al mantener un área de captación mayor y menos superficie no PV en los márgenes de las placas. Ello resulta en una mayor potencia en comparación a los PVs convencionales, además de tratarse de células de última generación de alta eficiencia. Además, estos permiten eliminar el efecto de puntos calientes que menguan el rendimiento de las células PVs, por efecto de sombreado. En caso de sombreado, la pérdida de producción es proporcional a la superficie afectada al disponer de micro-inversores, contrario a lo que sucede con los paneles tradicionales, que pueden descender desde un 33\% hasta casi la totalidad de producción, al conectarse estos en serie y con inversores compartidos centralizados. Los paneles PVs P19 provenientes de los fabricantes norteamericanos SunPower son productos que mantienen dimensiones de 1690x998x46 mm, entrega un total de $335 \mathrm{~W} / 37.50 \mathrm{~V}$, y posee un marco anodizado negro de clase 1 de aluminio de $40 \mathrm{~mm}$ (SunPower, 2020), destacado por su diseño uniforme totalmente negro que otorga elegancia y mejor adaptabilidad visual, alcanzando una eficiencia del 19.9\% (Sunfields - Europe, 2019).

Figura 8. Perspectiva con paneles sin contactos metálicos entre células y detalle constructivo
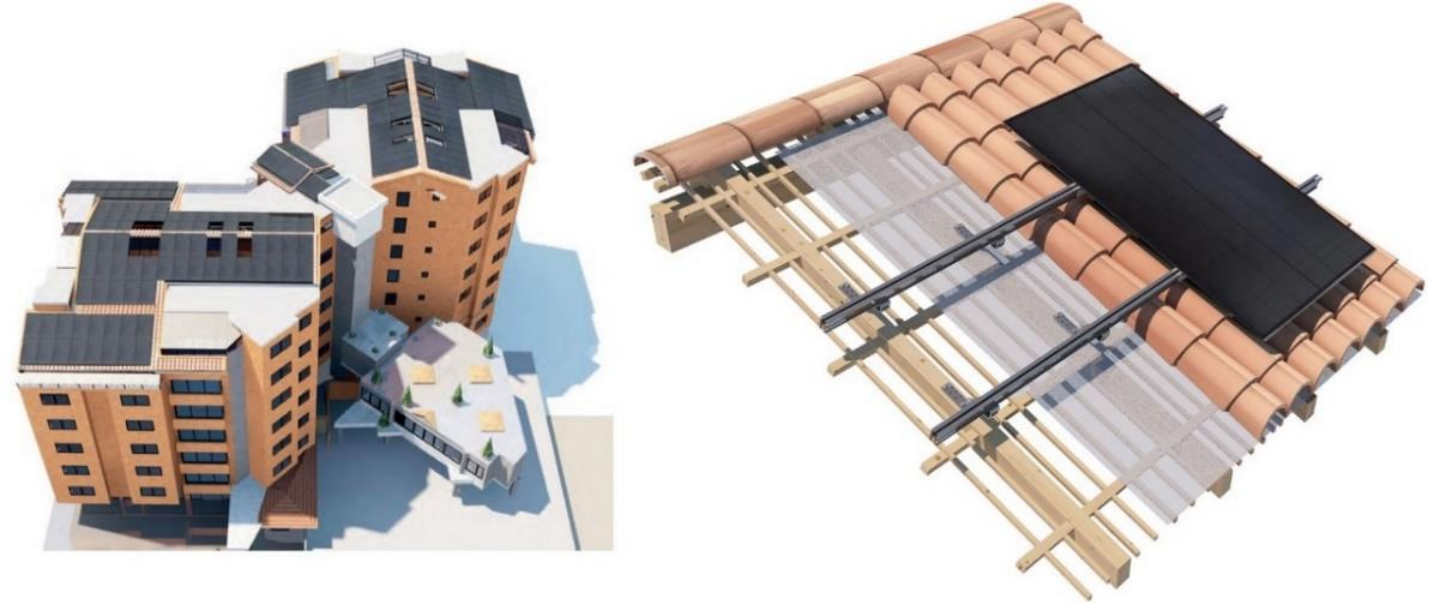

Fuente: Elaboración propia.

ACE, 15 (4.5) CC BY-ND 3.0 ES | UPC Barcelona, España | Capacidad e integración fotovoltaica en edificios 11 multifamiliares de mediana altura en la región ecuatorial andina. DOI: http://dx.doi.org/10.5821/ace.15.45.9307 
La edificación analizada abarca en la techumbre hasta 208 de estas unidades. La adaptabilidad a la cubierta se desarrolla de igual manera que la tecnología anteriormente mencionada; es decir, mediante perfiles que se acoplan sobre la cubierta existente y se anclan los paneles sobre estos, conservando la techumbre (Figura 8).

\subsection{Tejas PVs de silicio monocristalino (BIPV)}

Esta tecnología permite generar energía limpia de manera discreta. La principal característica es que desempeña un papel fundamental en cuanto al aspecto formal en la arquitectura debido a que quedan integradas en la envolvente de la edificación, mimetizándose con la techumbre tradicional (Martínez, 2016), siendo cobertura física, y generando un aspecto bastante favorable para su adaptación al ser apreciado de una manera compatible con la arquitectura de la ciudad.

Entre las ventajas se halla el gran parecido a las tejas, generando un impacto visual casi imperceptible (Inarquia, 2019). Mecánicamente, las tejas solares han alcanzado resistencias de hasta tres veces superiores a la teja clásica, debido a que están conformadas por tres capas: a) vidrio templado de alta resistencia que protege las células PVs y facilita el ingreso de luz; b) una película de color que actúa como una persiana según incide la luz; y, c) las células PVs de silicio (Shukla et al., 2016). Tesla Motors, por ejemplo, oferta cuatro modelos en el mercado: Teja Texturizada, Lisa, Pizarra y Toscana. Este último modelo Tesla es el considerado para aplicar en el análisis, el mismo que presenta dimensiones características de 184x365 mm, y una eficiencia del 12.0\% (Richardson, 2019); no obstante, por condiciones de instalación y forma de la teja, la superficie de instalación es la totalidad de la cubierta, con un resultado discreto sin exhibir sus células PVs desde cualquier ángulo de visión permitiendo crear un diseño óptimo e integrado. para que el tejado solar parezca una cubierta convencional.

Las tejas PVs reemplazan el tejado existente en la cubierta. Se adaptan a un sistema de machihembrado de madera de 3/4"x6" sobre la estructura de cubierta. Se instala una barrera de vapor con film de poliestireno similar a la técnica de tejado actual. Sobre la impermeabilización se fijan listones de madera de $1 \frac{1}{2} \mathbf{x}^{\prime}{ }^{1 / 4}$ " y tirantes de 2"x1". Se procede a colocar placas de poliestireno expandido con un espesor de $2 \mathrm{~cm}$ entre los listones y tirantes para nivelar los listones de sujeción y crear una superficie diáfana para la colocación de una segunda membrana impermeable de polietileno con cara de aluminio. Finalmente, se realiza la fijación de las tejas PVs (Figura 9).

Figura 9. Perspectiva con tejas PVs y detalle constructivo

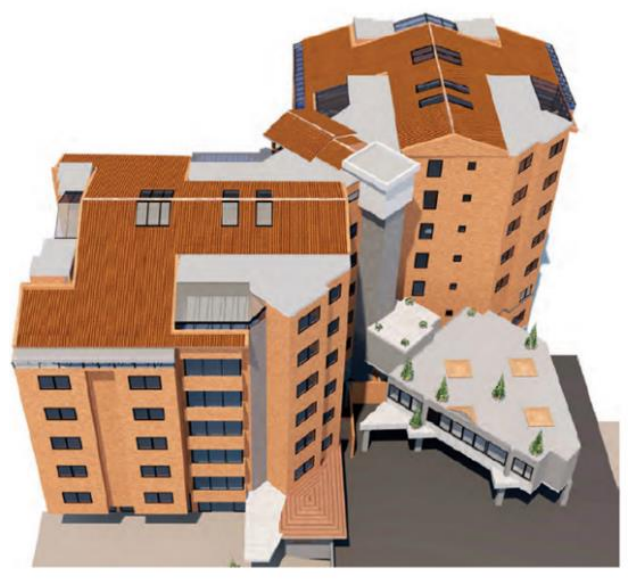

\section{LEYENDA}

1. Teja de caballete 2. Cumbrero

3. Machiembrado de madera 4. Listón de madera

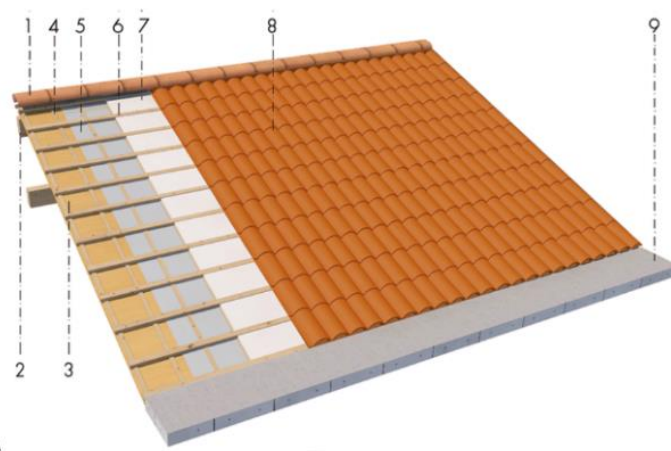

5. Membrana impermeable 8. Teja PV de poliestileno $\quad$ 9. Losa de hormigón 6. Clavadera de madera 10. Membrana 7. Placa de poliestireno impermeable de expandido

Fuente: Elaboración propia.

ACE, 15 (45) CC BY-ND 3.0 ES | UPC Barcelona, España | Capacidad e integración fotovoltaica en edificios multifamiliares de mediana altura en la región ecuatorial andina. DOI: http://dx.doi.org/10.5821/ace.15.45.9307 


\section{Simulación de rendimientos PVs}

Con base a las propuestas presentadas y modelo validado en SAM (Izquierdo et al., 2019) se obtuvo el rendimiento estimado de producción eléctrica por metro cuadrado de PV instalado para el año 2019. Las simulaciones se realizaron considerando las sombras arrojadas sobre la tecnología instalada y su eficiencia. La generación energética mensual alcanzada precisó que el mes de mayor producción es noviembre con 5262.79 kWh hasta 8727.46 kWh, y el de menor producción julio, con 3381.12 kWh hasta $4368.10 \mathrm{kWh}$, dependiendo de la tecnología utilizada (Figura 10). A partir de ello, se determina una variación del 19.8\% del valor promedio mensual identificado de 5548.78 kWh para los paneles convencionales, de $7112.58 \mathrm{kWh}$ para los paneles sin contactos metálicos y de $4295.02 \mathrm{kWh}$ para las tejas PVs.

Para el análisis de producción diaria-horaria y el correspondiente a días característicos-extremos, se profundizó en el estudio de los paneles tradicionales de silicio monocristalino, ya que de las otras dos tecnologías se cuenta únicamente con presunciones de rendimiento a partir de eficiencias publicadas. Es así que se determinó que la producción es 1.28 veces mayor en el caso de los paneles sin contactos metálicos entre células y 0.22 veces menor para las tejas.

Así, con base en los consumos registrados por los medidores cuarto horarios instalados en la edificación, se determinó una producción diaria promedio de $182.35 \mathrm{kWh}$. En las primeras horas del día y en consecuencia de la poca irradiación, se alcanzaron niveles de producción de 1.2 kWh y 4.4 kWh, llegando al pico al medio día con 35 kWh y descendiendo hasta 0 kWh al final de la tarde (18:00 horas). En confrontación con la demanda diaria determinada con antelación, se observó que en días laborables existe una sobreproducción promedio de $37.6 \%$ y en el fin de semana se evidencia un excedente promedio de $103.04 \%$.

Figura 10. Demanda vs Producción energética

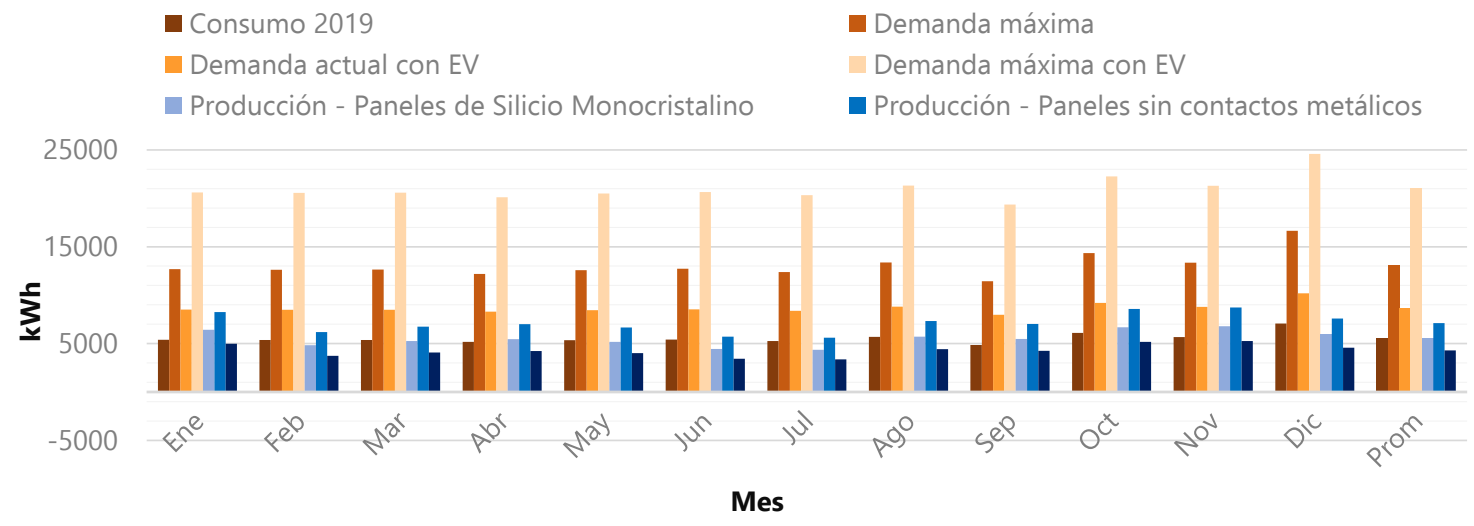

Fuente: Elaboración propia a partir de la información proporcionada por la Empresa Eléctrica Regional Centro Sur y simulaciones de producción fotovoltaica en SAM.

De igual manera, al comparar las horas de mayor consumo durante el día (09:00 a 11:00 h) que presentan valores de requerimiento energético entre $7.62 \mathrm{kWh}$ y $7.06 \mathrm{kWh}$, frente al potencial de generación en esas mismas horas, se observó una sobreproducción del triple de lo requerido, al encontrarse un valor promedio de $21.97 \mathrm{kWh}$. Si bien las demandas altas coinciden con las horas de mayor insolación, es esperable que haya excedentes habitualmente. En la Figura 11 se muestra la producción diaria total anual del edificio acorde a la tecnología PV empleada, pudiendo apreciarse el rendimiento y aporte energético. Se alcanza la máxima generación diaria de hasta $376.69 \mathrm{kWh}$ con las placas negras sin contactos metálicos, alrededor de 293.46 kWh con las placas convencionales de silicio y cerca de $227.15 \mathrm{kWh}$ con las tejas PVs.

ACE, 15 (4.5) CC BY-ND 3.0 ES | UPC Barcelona, España | Capacidad e integración fotovoltaica en edificios 13 multifamiliares de mediana altura en la región ecuatorial andina. DOI: http://dx.doi.org/10.5821/ace.15.45.9307 
Figura 11. Producción diaria del edificio, según tecnología PV

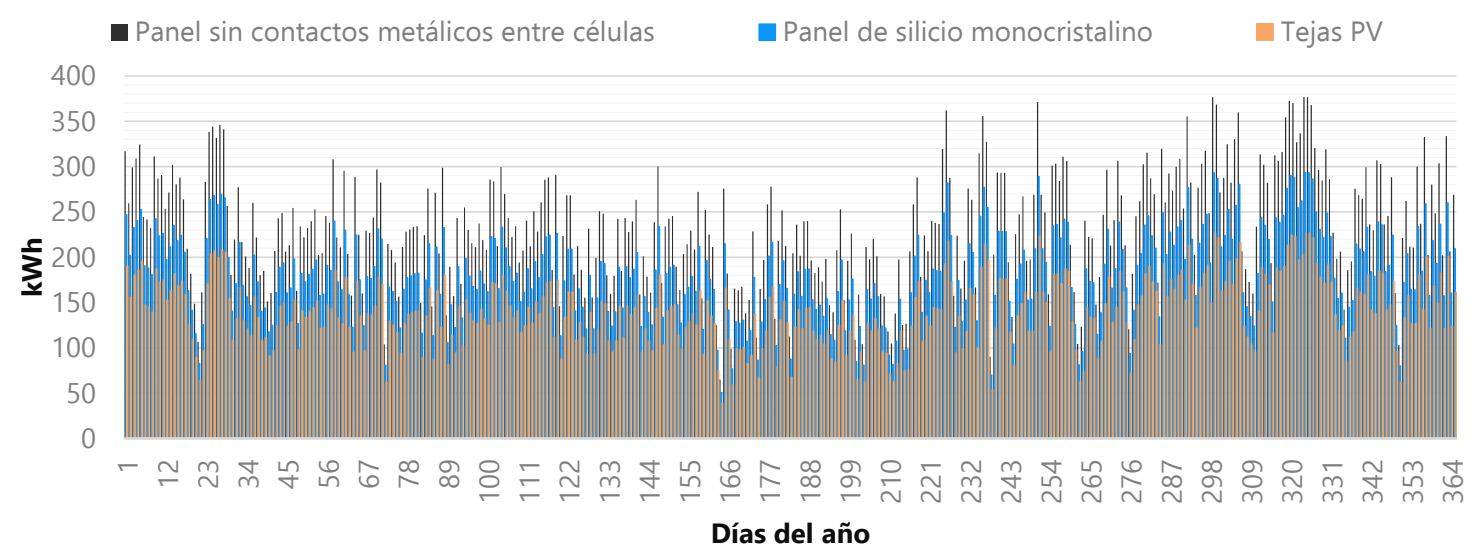

Fuente: Elaboración propia a partir de los datos de producción energética obtenidos mediante las simulaciones.

Con la producción determinada en la totalidad de techumbre con placas tradicionales, se analiza el escenario para los días de máxima y mínima irradiación correspondiente al 26 de octubre y 13 de junio, respectivamente. El análisis se complementa con los casos de días promedio del mes de máxima y mínima irradiación, noviembre y julio; y, por último, con el día promedio de producción. Estos casos entran en confrontación con los días de demanda mínima, máxima y promedio. En la Figura 12 se muestran las curvas horarias en comparación con el supuesto de Ocupación de usuarios Actual (OA) y Ocupación de usuarios Total (OT), evidenciando la elevada producción al medio día con excedentes en la mayoría de los casos.

Figura 12. Producción vs consumo eléctrico horario en días extremos

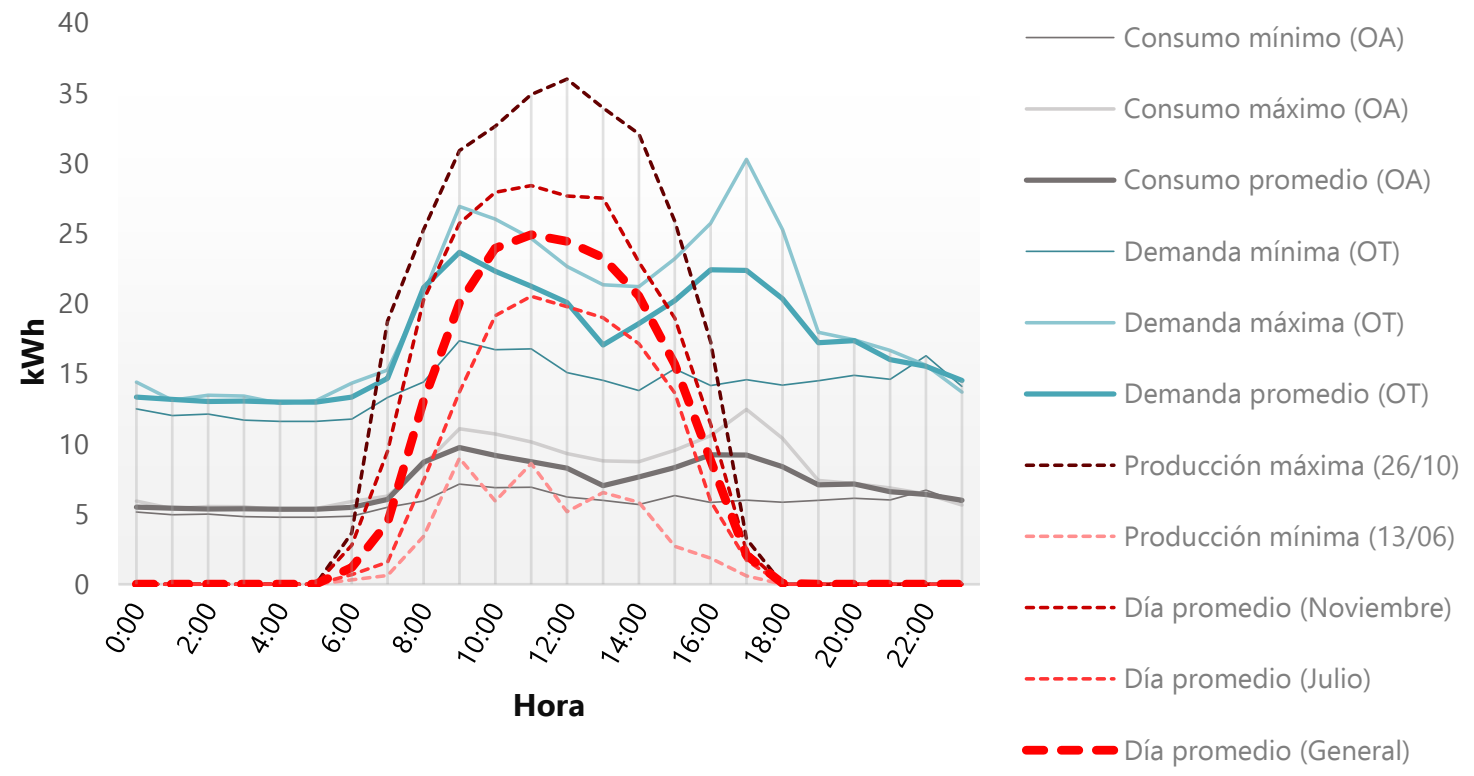

Fuente: Elaboración propia con base en la información registrada por los medidores cuarto-horarios y simulaciones de producción PV en SAM.

En la Tabla 2 se muestra un resumen de características por tecnología PV como: el balance anual de la producción, la potencia nominal y pico, el porcentaje de autoabastecimiento, costo, entre otros aspectos.

ACE, 15 (4.5) CC BY-ND 3.0 ES | UPC Barcelona, España | Capacidad e integración fotovoltaica en edificios 14 multifamiliares de mediana altura en la región ecuatorial andina. DOI: http://dx.doi.org/10.5821/ace.15.45.9307 
Tabla 2. Resumen de características por tecnología PV

\begin{tabular}{|c|c|c|c|}
\hline Tecnología & $\begin{array}{l}\text { Panel de silicio } \\
\text { monocristalino }\end{array}$ & $\begin{array}{c}\text { Panel sin contactos } \\
\text { metálicos }\end{array}$ & Tejas PVs \\
\hline Producción (kWh/año) & 66585.31 & 85470.96 & 51540.27 \\
\hline Producción (kWh/m²/año) & 196.77 & 243.63 & 135.73 \\
\hline Cantidad $\left(\mathrm{m}^{2}\right)$ & 338.4 & 350.82 & 379.72 \\
\hline Potencia pico (kWp) & 59.28 & 69.68 & 42.40 \\
\hline Costo por W instalado (\$) & 1.31 & 1.24 & 2.86 \\
\hline Abastecimiento (d. actual) & $99.72 \%$ & $128.00 \%$ & $77.18 \%$ \\
\hline Abastecimiento (d. máxima) & $46.24 \%$ & $59.35 \%$ & $35.79 \%$ \\
\hline Abastecimiento (d. actual con EV) & $63.94 \%$ & $82.07 \%$ & $49.49 \%$ \\
\hline Abastecimiento (d. máxima con EV) & $27.85 \%$ & $35.74 \%$ & $21.55 \%$ \\
\hline Costo aproximado & $\$ 107863.86$ & $\$ 80294.61$ & $\$ 118570.59$ \\
\hline Consumo actual (kWh/año) & & 66775.62 & \\
\hline Demanda máxima (kWh/año) & & 144012.01 & \\
\hline Demanda actual con EVs (kWh/año) & & 104141.52 & \\
\hline Demanda máxima con EVs (kWh/año) & & 239125.21 & \\
\hline
\end{tabular}

Fuente: Elaboración propia.

\section{Implicaciones arquitectónicas}

En este apartado se analizan las implicaciones arquitectónicas de la incorporación de las tecnologías BAPV y BIPV en la edificación mixta, considerando niveles de integración propuestos acorde a cada tecnología. Se aporta con una apreciación general de la incorporación de estos productos en la construcción, la cual servirá como punto de partida para investigaciones más específicas sobre el impacto urbano-arquitectónico causado por las tecnologías solares. Este tipo de análisis requiere de un estudio profundo que permita precisar las distintas percepciones de la sociedad; por lo tanto, se sugiere revisar la metodología planteada por las autoras Sánchez, Vidal y Pastor en la investigación "Aesthetic impact of solar energy systems" (Sánchez et al., 2018), en la cual se evalúa la amplia gama de factores que se encuentran en la teoría y la falta de consenso sobre cómo aplicarlos para analizar el impacto de la implementación de sistemas solares

La principal contribución del estudio es el establecimiento de los factores más influyentes basados en la literatura revisada, que, coincidiendo con lo expuesto, son los más complejos: la visibilidad (o tamaño) y el grado de integración. Como resultado, las autoras proponen un marco metodológico, para evaluar el impacto estético dividido en tres niveles o sub-impactos: 1) uso del suelo, que depende del tamaño; 2) sistema de energía solar, que está en relación con la visibilidad; y. 3) el grado de integración y el deslumbramiento, que igualmente depende de la visibilidad (Sánchez et al., 2018). El análisis de estas características es un complemento a lo presentado, pero se trata de un estudio en sí mismo.

\subsection{Niveles de integración arquitectónica de las tecnologías}

Se realizó un análisis de la implicancia visual generada por los PVs desde la perspectiva arquitectónica y urbanística con base en los criterios de integración de energía solar activa en arquitectura de los autores Zalamea \& Quesada, (2017) con respecto a las conceptualizaciones teóricas fundamentales propuestas por Kaan \& Reijenga, (2004).

Los paneles de silicio monocristalino de 60 celdas y las placas sin contactos metálicos entre células como alternativas BAPV, han sido categorizadas como tecnologías con Nivel 1 - Formal de inclusión, considerando que se encuentran únicamente superpuestos en la cubierta, de forma paralela y coplanar con cada faldón, sin pasar a formar parte de la cubierta como un material de revestimiento. Estos, con la estructura de las placas PVs fijada al envigado del edificio, se encuentran ligeramente 


\section{ACE Architecture, City and Environment}

E-ISSN $1886-4805$

elevados sobre la teja, lo que, en conjunto con su color azul característico (placas convencionales) los vuelve más notorios (Figura 13). En cambio, la segunda tecnología se presenta como una propuesta alternativa a la vista desde la perspectiva estética, aunque desde distancias cercanas a la calle ni siquiera es perceptible. La uniformidad generada por el color negro no es especialmente impactante, pero contrasta con el medio respecto a la mayor parte de los otros techos (Figura 14).

Por último, al ser las tejas PVs un sistema BIPV, tienen una connotación en la expresividad al mimetizarse en un Nivel 3 - Expresión. Esta tecnología está completamente integrada, ya que aprovecha toda la techumbre para su incorporación, que por sus dimensiones generan visualmente un aspecto nada notorio respecto al resto de cubiertas y mamposterías (Figura 15).

En referencia a los niveles de integración propuestos por los autores, en esta edificación no se puede plantear el Nivel 4 - Multiplicidad, debido a que, para ello, el diseño del edificio o una eventual intervención debería potenciar la captación solar y lograr que la expresión aporte significativamente la estética del edificio, lo cual no se considera como una posibilidad en esta edificación ya existente.

Figura 13. Perspectiva noreste con paneles de silicio monocristalino

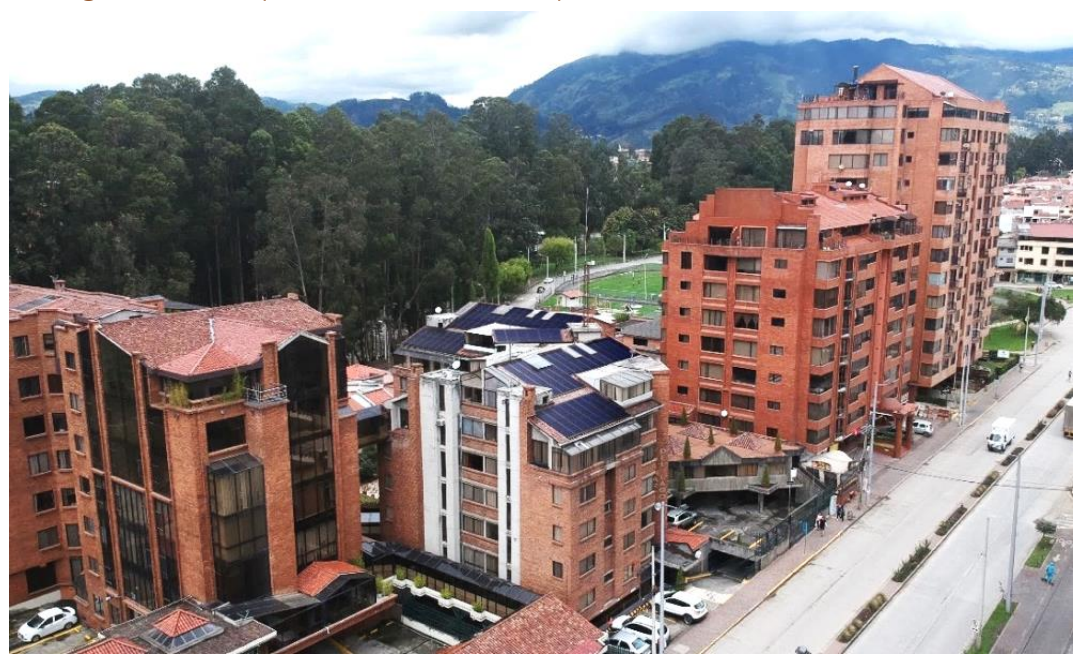

Fuente: Autores.

Figura 14. Perspectiva suroeste con paneles sin contactos metálicos entre células

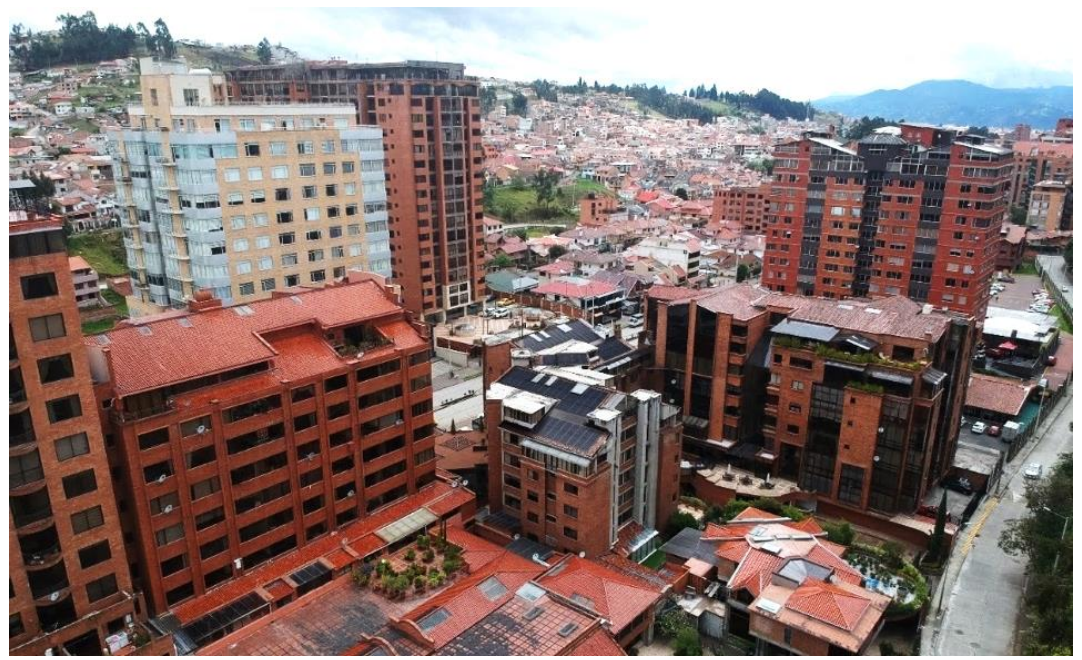

Fuente: Autores.

ACE, 15 (4.5) CC BY-ND 3.0 ES | UPC Barcelona, España | Capacidad e integración fotovoltaica en edificios 16 multifamiliares de mediana altura en la región ecuatorial andina. DOI: http://dx.doi.org/10.5821/ace.15.45.9307 


\section{ACE Architecture, City and Environment}

E-ISSN $1886-4805$

Figura 15. Perspectiva frontal con tejas PVs

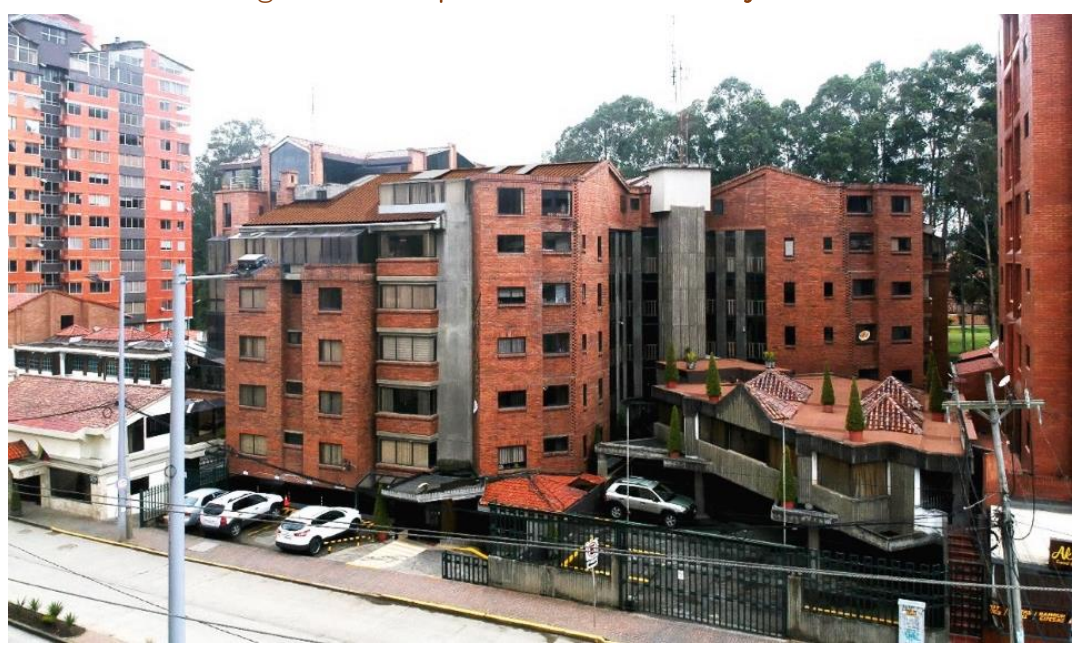

Fuente: Autores.

\subsection{Impacto visual al entorno inmediato}

Se determinó que el impacto por la incorporación de las tecnologías a vista del observador resultó, desde la mayoría de los ángulos, prácticamente imperceptible para los transeúntes a nivel de la calle. Esto, como consecuencia del número de pisos de la construcción. Por ello, en la Figura 16 se exponen las perspectivas en que se observan los PVs desde nivel de suelo. La materialidad se evidencia desde lugares apartados, entre cuarenta y cien metros de distancia.

Figura 16. Impacto visual por la incorporación de tecnologías PVs
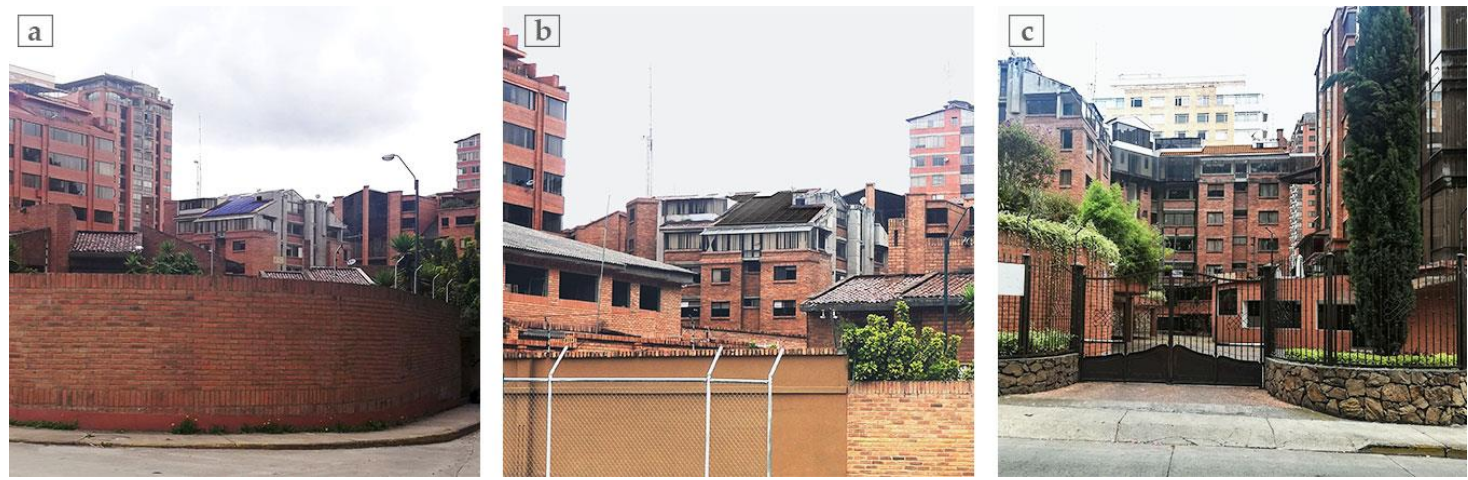

(a) Integración de paneles de silicio monocristalino; (b) Integración de paneles sin contactos metálicos; (c) Integración de Tejas PVs.

Fuente: Autores.

Por otro lado, desde perspectivas aéreas y para aquellas personas que habitan en los edificios aledaños, el impacto visual es evidente en mayor o menor grado según la tecnología empleada. No obstante, se observan otros tipos de instalaciones que afectan la expresión de los techos como instalaciones de antenas, pararrayos, bloques de elevadores o lucernarios (Figura 13 y Figura 15). Sin embargo, el impacto ambiental puede ser aún más significativo en instalaciones de huertos PVs emplazados en zonas campestres y agrestes, puesto que la alteración no es solo estética sino con implicancias y afecciones silvestres (Poggi et al., 2018). En paisajes urbanos el impacto desde el punto de vista ambiental es mucho menor, por lo que la incorporación de tecnologías solares in situ resulta idónea.

ACE, 15 (4.5) CC BY-ND 3.0 ES | UPC Barcelona, España | Capacidad e integración fotovoltaica en edificios 17 multifamiliares de mediana altura en la región ecuatorial andina. DOI: http://dx.doi.org/10.5821/ace.15.45.9307 


\section{Análisis financiero}

La instalación de un sistema de captación solar activa debe ser observado desde la perspectiva económica, evaluando su practicidad desde el análisis de la Tasa Interna de Retorno (TIR) y el Valor Actual Neto (VAN). A solicitud de la administración del edificio se realizó un análisis para valorar la factibilidad financiera, considerando factores como: el costo medio de energía subsidiada facturada como consumo residencial de $\$ 0.093 / \mathrm{kWh}$, el precio real de la electricidad sin subsidio, que se estima en alrededor de \$0.16 el kWh según información de la Corporación Eléctrica del Ecuador (CELEC, 2014), y la tasa de interés pasiva bancaria que en la actualidad está en 8.68\%, conforme al Banco Central del Ecuador (ARCONEL, 2018a). La evaluación financiera de las tecnologías se realizó con precios al mercado estadounidense con la finalidad de analizarlas en condiciones similares, puesto que los productos analizados aún no se comercializan en el Ecuador, pero se extrapolan luego a valores factibles en el mercado local. Se tomó como punto de partida el precio de la tecnología existente tanto en el mercado local (ProViento S.A. Energías Renovables Ecuador, 2019) como internacional (paneles tradicionales), y se determinó que esta última llega a costar hasta un 60\% más en Ecuador. Con base en estos criterios, se desarrollaron tres escenarios en los cuales se comparó cada tecnología con su costo de inversión y producción eléctrica.

Tabla 3. Costos de inversión tecnologías BIPV - BAPV

\begin{tabular}{|c|c|c|c|}
\hline Tecnología & $\begin{array}{l}\text { Panel de silicio } \\
\text { monocristalino }\end{array}$ & $\begin{array}{c}\text { Panel sin contactos } \\
\text { metálicos }\end{array}$ & Tejas PVs \\
\hline Costo tecnología PV x unidad $(\$)$ & $87.62 / \mathrm{m}^{2}$ & $99.15 / \mathrm{m}^{2}$ & $183.51 / \mathrm{m}^{2}$ \\
\hline Costo Inversores $x$ unidad $(\$)$ & 2018.42 & 2018.42 & 2018.42 \\
\hline Costo total tecnología PV (\$) & 29649.73 & 34782.81 & 69682.42 \\
\hline Costo total de 8 inversores $(\$)$ & 16147.36 & 16147.36 & 16147.36 \\
\hline Subtotal (PV + inversores) (\$) & 45797.09 & 509030.17 & 85829.78 \\
\hline a. Equipos y materiales & 60452.16 & 67227.83 & 94412.75 \\
\hline b. Instalación (15\%) & 9067.82 & 10084.17 & 14161.91 \\
\hline c. Subtotal costos directos $(a+b)$ & 69519.99 & 77312.00 & 108574.67 \\
\hline d. $\quad$ Costo indirecto $(12 \%)$ & 8342.40 & 9277.44 & 13028.96 \\
\hline e. Inversión total sin IVA (\$) & 77862.38 & 86589.44 & 121603.63 \\
\hline
\end{tabular}

Fuente: Elaboración propia.

En el primer escenario con paneles convencionales de silicio monocristalino, se asume la instalación de 208 PVs, con equipos y materiales. Se consideró el mantenimiento de las instalaciones durante 25 años alcanzando un valor aproximado de \$77862.38, detallado en la Tabla 3. La implementación de la tecnología con el subsidio eléctrico permitiría un ahorro de alrededor de $\$ 6758.41$ al año, con un VAN positivo de $\$ 67098.32$ y una TIR de -0.25\%, cubriéndose la inversión en 13 años (Figura 17). Además, se realizó el análisis considerando el costo real de electricidad, obteniendo un VAN de $\$ 109865.44$, una TIR de 4.50\% y recuperando la inversión en 8 años. Al comparar el costo real de estos paneles existentes a nivel local e incrementando su valor $60 \%$, el panorama es completamente diferente, puesto que el tiempo de recuperación aumenta notablemente. Cabe mencionar que para los análisis de las tres tecnologías se consideró el reemplazo de inversores dentro de un período de 12 años; es decir, se consideró un recambio debido a su vida útil y costos de mantenimiento (Electric Power Research Institute, 2015).

Para el segundo escenario con los paneles PVs sin contactos metálicos entre células, se tomaron como referencia los precios ofertados a nivel internacional para la instalación de 208 placas, dado que el producto no se oferta localmente. Se consideró el mantenimiento de la instalación durante su vida útil dando un costo de aproximadamente \$86589.44 (Tabla 3). Se calculó que el ahorro anual para la edificación evitaría el pago a la comercializadora eléctrica alrededor de \$8675.30 al año. Con el precio de la energía subsidiada, el VAN resultante positivo fue de \$106865.81, con una TIR de 3.11\%, pudiendo recuperar la inversión en 9 años. En tanto que, al no tomar en cuenta el subsidio, se tuvo 
un VAN de \$159003.91 una TIR de 7.57\% y un periodo de amortización de 6 años (Ver Figura 17), lo cual evidencia una rentabilidad muy alta.

En el tercer escenario se evaluó la rentabilidad de las Tejas PVs $\left(379.72 \mathrm{~m}^{2}\right)$. De igual manera que en el caso anterior, se consideraron precios internacionales que arrojaron un coste de \$121603.63 (Tabla 3). De esta manera la edificación ahorraría alrededor de $\$ 5231.34$ anualmente. Con el precio subsidiado de la energía facturada, el VAN resultó positivo $\$ 74937.49$ con una TIR de $-3.02 \%$, con un período de recuperación de la inversión de 19 años; es decir, en absoluto conveniente. Sin embargo, al no considerar los subsidios, se estimó un VAN de \$106377.55, una TIR de -0.12\% y la recuperación de la inversión se redujo a 13 años (Figura 17).

Figura 17. Flujo de caja de las tecnologías BAPV y BIPV con y sin subsidio (Período de amortización)

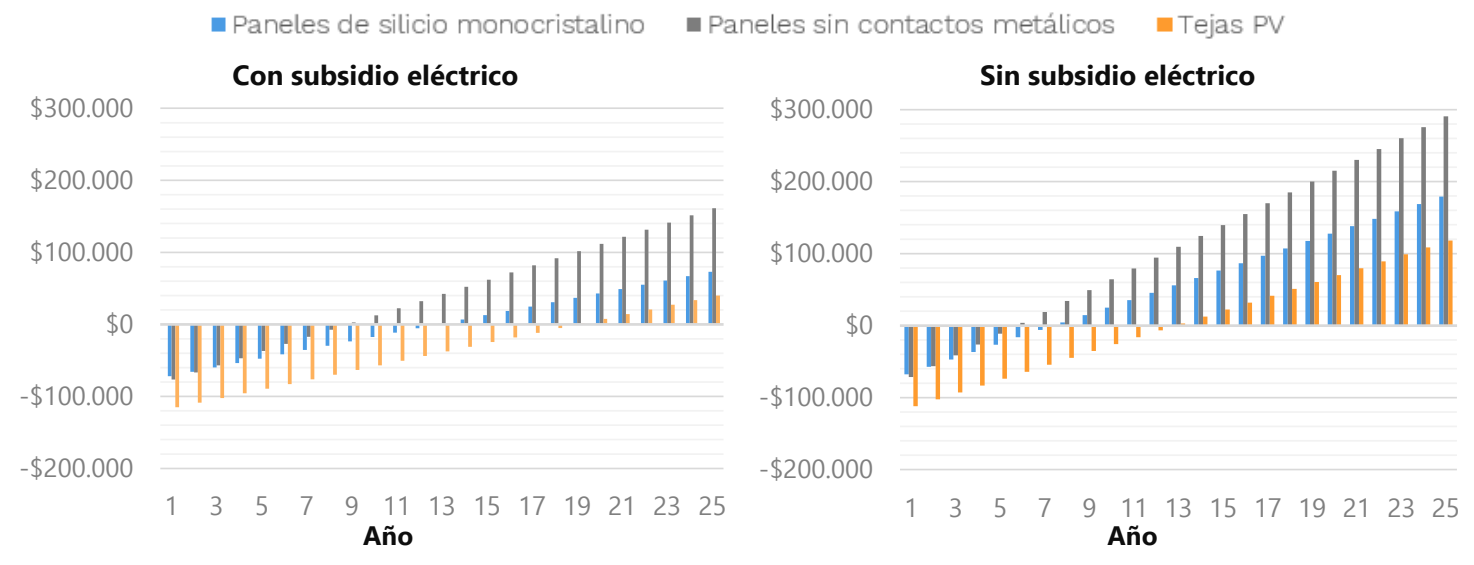

Fuente: Elaboración propia.

\section{Discusión y conclusiones}

El propósito de este trabajo fue establecer el uso de captación solar a través de tecnología PV en un edificio de uso mixto. Se tomó como caso de estudio una edificación existente para inferir las condiciones generales y el aporte que tendría el uso de estas alternativas de autoaprovisionamiento energético. Además, el análisis permitió revisar la integración arquitectónica urbanística de la tecnología PV. En este contexto, el estudio refleja que los edificios de uso residencial-comercial de la ciudad de Cuenca son un soporte propicio para resolver la demanda energética desde fuentes renovables, para revertir el consumo y reducir la combustión urbana.

Los resultados evidenciaron que el consumo energético mensual promedio del edificio en el año 2019 fue de 5564.64 kWh bajo las condiciones de ocupación existente y se estimó alcanzaría una demanda mensual promedio de 12001.00 kWh con la ocupación para la que fue diseñado el condominio.

El análisis de sombras muestra la ventaja resultante de la cercanía a la línea ecuatorial y por ende una gran altura solar. Tomando en cuenta el nivel de irradiación solar de Cuenca (1600 kWh/m² año) y la superficie total de techumbre de la edificación $\left(651.54 \mathrm{~m}^{2}\right)$, se determina que podrían existir alrededor de 1042.50 MWh de irradiación solar al año, siendo particular la condición de que todos los faldones de techumbre reciben irradiación directa, con sombras en horas del día sobre todo vespertinas y que implican pérdidas menores. Al compararse con proyectos similares encontrados en países como Suiza, Finlandia, Austria, Italia o Alemania, expuestos en la Tabla 1; se establece que en Ecuador el potencial de energía PV oscila entre los 876 - 1753 kWh/kWp/año, seguido por Italia con 949 - 1680 kWh/kWp/año y al final Finlandia, con un índice de 949 -1168 kWh/kWp/año (Solargis, 
2017). Se demuestra además la gran ventaja de la latitud ecuatorial que permite la captación de irradiación en faldones orientados en cualquier dirección.

Es relevante mencionar que hasta lo que se pudo examinar, no se ha encontrado literatura con estudios similares en climas ecuatoriales y en altura. Sin embargo, una investigación bastante cercana al tema desarrollado se presentó en Brasil. El trabajo analizó el potencial de 7 tecnologías BIPV implementadas en un típico edificio residencial simulado en tres ciudades diferentes. Los resultados revelaron un potencial interesante para la generación PV incluso en superficies verticales en sitios de baja latitud generando, durante el 30\% del año, más energía que la demanda de la construcción, posibilitando suministrar energía a la red pública de electricidad (Ordenes et al., 2007). Por otro lado, se hallaron estudios que tratan el potencial PV integrado en edificios comerciales, en aeropuertos (Rüther \& Braun, 2009), rendimiento PV para la electrificación rural (Feron \& Cordero, 2018; RosasFlores et al., 2019), entre otros. No obstante, estos últimos fueron enfocados al potencial energético, tratándose en otras investigaciones el tema de la integración arquitectónica (Sánchez et al., 2018). Así también, se evidenció una mayor cantidad de análisis de rendimientos PVs en climas mediterráneos.

Mediante las simulaciones de producción, se revela que los paneles convencionales de silicio monocristalino desplegados en el caso de estudio con una potencia pico de $59.28 \mathrm{kWp}$, generan $66585.31 \mathrm{kWh} /$ año rindiendo $196.77 \mathrm{kWh} / \mathrm{m}^{2} /$ año, logrando abastecer en $99.72 \%$ y $46.24 \%$, alcanzando el estándar Net-Zero bajo la ocupación actual, es decir, el edificio consume poca energía comparado con el consumo promedio de edificaciones similares además de reducir la cantidad de energía requerida para su funcionamiento mediante fuentes renovables (Usón Guardiola et al., 2014). Los cálculos estimados muestran que el edificio admitiría un total de 208 PVs con una potencia nominal de 285 W y eficiencia del $15.5 \%$.

En cambio, los paneles sin contactos metálicos entre células con 208 unidades de alto rendimiento monocristalino de 335 W con una eficiencia del 19.9\% (Sunfields - Europe, 2019) y una potencia pico instalada de $69.68 \mathrm{kWp}$, alcanzan una producción de $85470.96 \mathrm{kWh} /$ año y $243.63 \mathrm{kWh} / \mathrm{m}^{2} / a n ̃ o$. Con ello, se logra un autoabastecimiento de $128.00 \%$ en el caso de la demanda actual y de $59.35 \%$ con respecto a la demanda máxima esperada, brindando hasta un 28.28\% adicional de electricidad con relación a las placas tradicionales (Sunfields - Europe, 2019).

En cuanto a las tejas PVs, con 5654 unidades mantiene una mayor ocupación por su formato pero menor eficiencia 12\% (Richardson, 2019). Mediante una potencia pico instalada de $42.40 \mathrm{kWp}$ se logra generar aproximadamente 51540.27 kWh/año y $135.73 \mathrm{kWh} / \mathrm{m}^{2} / a n ̃ o$, alcanzando porcentajes de autoabastecimiento energético de $77.18 \%$ y 35.79\%, de la demanda actual y máxima, respectivamente.

Financieramente, se analiza la generación eléctrica con base en una inversión en el mercado internacional, con lo cual se puede predeterminar una amortización de inversión. Se vio de importancia el considerar el escenario de electricidad no subvencionada, para presentar un análisis alternativo para un eventual redireccionamiento de subsidios. Así, se analizaron los tres sistemas determinando que, al mantener los costos de subsidio actuales a la electricidad de la red, la tecnología que mejor periodo de amortización representa son los paneles sin contactos metálicos con un tiempo de recuperación de 9 años frente a los 13 de los paneles tradicionales y los 19 años de las tejas PVs denotando que, bajo condiciones actuales, resulta poco o no atractiva la inversión PV. Bajo el escenario de eliminación de subsidios a la energía de la red, los paneles sin contactos metálicos siguen liderando en menor tiempo de amortización con apenas 6 años de recuperación de inversión lo que evidencia una rentabilidad alta; en comparación con los paneles tradicionales y tejas $\mathrm{PV}$ s, que recuperan su inversión en 8 y 13 años, respectivamente.

Los estudios se realizaron con placas de 60 celdas con potencia de hasta $330 \mathrm{~W}$, pero actualmente se están fabricando productos de más de $800 \mathrm{~W}$ que se prevén prontamente disponibles en el

ACE, 15 (4.5) CC BY-ND 3.0 ES | UPC Barcelona, España | Capacidad e integración fotovoltaica en edificios 20 multifamiliares de mediana altura en la región ecuatorial andina. DOI: http://dx.doi.org/10.5821/ace.15.45.9307 
mercado local, lo que sugiere que, de todos modos, se convertirán en tecnologías atractivas en el corto plazo, pero con menor incidencia respecto a lo que sucedería con apoyo estatal decidido. Esto supone que, si bien estas tecnologías ya denotan cierta factibilidad, especialmente aquella de alta eficiencia, la masificación en el mercado es necesaria para una mayor reducción de los costos. A pesar de estas estimaciones poco alentadoras, se evidencia que, de trasladarse la alta inversión estatal dirigida últimamente solo a los proyectos hidroeléctricos estatales, los cuales tuvieron una inversión inicial de aproximadamente 4700 millones de dólares hasta el año 2016 (Urgilés et al., 2015), de destinarse recursos similares a la microgeneración se puede tornar una opción altamente competitiva y serían recursos que provoquen puestos de trabajo locales además de promover la diversificación de fuentes energéticas y la conversión de demandas a electricidad limpia.

Desde las implicaciones arquitectónicas de la incorporación de las tecnologías BAPV y BIPV en el caso analizado, y desde las visuales obtenidas a nivel de transeúnte urbano, se determina que los PVs son poco visibles, pasando prácticamente desapercibidos e incluso otras instalaciones en las cubiertas resultan más contrastantes. Sin embargo, desde edificaciones aledañas y perspectivas aéreas, la afección al entorno se da en mayor o menor grado acorde a la tecnología empleada. Además, se aprecia que otras instalaciones y redes eléctricas y electrónicas, antenas, tanques o lucernarios implican acaso mayor afectación.

Considerando la interacción de los datos calculados con el factor de emisión de 300.2 gCO $/ \mathrm{kWh}$ publicado por el Operador Nacional de Electricidad (CENACE), ente técnico responsable del cálculo de las emisiones de $\mathrm{CO}_{2}$ del Sistema Nacional Interconectado del Ecuador (CENACE, 2018), se estima que en un año se evitaría la emisión de $19456 \mathrm{~kg}$ de $\mathrm{CO}_{2}$ si se toma en cuenta la demanda actual, y $47274 \mathrm{~kg}$ de $\mathrm{CO}_{2}$ si se considera la demanda máxima. En cambio, en un escenario futuro, y a lo largo de la vida útil de los paneles PVs (25 años), se conseguiría evitar la emisión de hasta 1'181.842 kg de $\mathrm{CO}_{2}$. Estos resultados son positivos si se los compara con los datos de las edificaciones analizadas en Europa (Tabla 1), que demuestran una reducción de entre $16.000 \mathrm{~kg}$ y $100.000 \mathrm{~kg}$ de $\mathrm{CO}_{2}$, considerando un año de producción de energía mediante PV. Por tanto, el edificio de la ciudad de Cuenca estaría al nivel de los casos de estudio internacionales en cuanto a la reducción de emisiones.

Finalmente, se podría considerar que la integración de sistemas PVs como fuente de energía eléctrica autosuficiente y autónoma en edificios multifamiliares emplazados en latitudes ecuatoriales, es viable, por las características constructivas y técnicas con las que actualmente se encuentran en el mercado los módulos solares. Como complemento y trabajo futuro, es necesaria la definición de aspectos de la instalación puntual de las redes internas del condominio y condiciones normativas respecto a la distribución de las ganancias solares y costos de instalación entre copropietarios. Además, se requieren estudiar metodologías que permitan determinar las implicancias ambientales urbanas frente a la dramática reducción de costos de la tecnología PV, el incremento de equipamiento y transporte eléctrico y la necesidad de reducir la combustión de los centros urbanos. La incorporación de autogeneración eléctrica se muestra como una alternativa idónea, pero tiene que ser impulsada desde políticas estatales y financieras.

\section{Agradecimientos}

Este trabajo es parte del proyecto de investigación: “Abastecimiento energético renovable desde recursos endógenos, en ciudades de países en vías de desarrollo en el marco del metabolismo urbano. Caso de Estudio Cuenca, Ecuador". Se agradece a la Dirección de Investigación de la Universidad de Cuenca (DIUC), Centro de Investigación de la Facultad de Arquitectura (CINA) de la Universidad de Cuenca y la Universidad Politécnica Salesiana por el apoyo brindado. Al Condominio Pinar del Lago por la información facilitada para el desarrollo de la presente investigación y la Empresa Eléctrica Regional Centro Sur, por las facilidades prestadas en el montaje de equipos de medición.

ACE, 15 (4.5) CC BY-ND 3.0 ES | UPC Barcelona, España | Capacidad e integración fotovoltaica en edificios 21 multifamiliares de mediana altura en la región ecuatorial andina. DOI: http://dx.doi.org/10.5821/ace.15.45.9307 


\section{Autoría}

El primer y segundo autor efectuaron la conceptualización, diseño, levantamiento, procesamiento y escritura de la investigación, el tercer autor se ha encargado del diseño de la metodología y ha asesorado toda la investigación y el cuarto autor realizó la revisión de la propuesta eléctrica y del artículo.

Conflicto de intereses: Los autores declaran que no hay conflicto de intereses.

\section{Bibliografía}

Aguacil, S., Lufkin, S., \& Rey, E. (2017). Integrated design strategies for renovation projects with BuildingIntegrated Photovoltaics towards Low-Carbon Buildings: Two comparative case studies in Neuchâtel (Switzerland). 33rd PLEA Conference Proceedings PLEA Conference Proceedings: Design to Thrive, II(July), $\quad$ 3000-3007. Recuperado de https://www.researchgate.net/publication/318275186 Integrated design strategies for renovation p rojects_with_Building-Integrated_Photovoltaics_towards_Low-

Carbon_Buildings_Two_comparative_case_studies_in_Neuchatel_switzerland

ARCONEL. (2018a). Estadistica anual y multianual del servicio eléctrico ecuatoriano. Recuperado de https://www.regulacionelectrica.gob.ec/wp-

content/uploads/downloads/2019/08/EstadísticaAnualMultianual2018.pdf

ARCONEL. (2018b). Resolución Nro. ARCONEL-042/18. Recuperado de https://www.regulacionelectrica.gob.ec/wp-content/uploads/downloads/2019/03/042-18.pdf

Baquero L, M. T., \& Quesada M., F. (2016). Energy efficiency in Cuenca's residential sector, Ecuador. MASKANA, 7(2), 147-165. DOI: https://doi.org/https://doi.org/10.18537/mskn.07.02.11

Baur-Gschier, D. U., Stückler, D. H., \& Mayrhofer, I. (2014). The Nearly Zero-Energy Building Building for the Future Contents. LandesEnergieVerein Steiermark project team. Recuperado de https://www.eastmk.at/documents/20181/25550/NZEB Broschuere englisch WEB.pdf/78737a82-4b16-4e24-a8c8$\underline{36 d 0501 \mathrm{befd} 6}$

CELEC. (2014). CELEC EP -Vigente el mecanismo de subsidio eléctrico. Recuperado de https://www.celec.gob.ec/termopichincha/

CENACE. (2018). Informe del Factor de Emisión de CO2 2018. 14. Recuperado de http://www.cenace.gob.ec/wp-content/uploads/downloads/2020/04/Informe-Factor-de-CO2Sector-Eléctrico-2018.pdf

Climate-Data.org. (2014). Clima CUENCA: Temperatura, Climograma y Tabla climática para CUENCA Climate-Data.org. Recuperado de https://es.climate-data.org/america-del-sur/ecuador/provinciadel-azuay/cuenca-875185/

Electric Power Research Institute. (2015). Budgeting for Solar PV Plant O\&M: Practices \& Pricing. Recuperado de https://www.epri.com/

Erhorn, H., \& Erhorn-Kluttig, H. (2014). Selected examples of Nearly Zero-Energy Buildings Detailed Report. September, 74. DOI: https://doi.org/10.1016/j.egypro.2014.11.1011 
Feron, S., \& Cordero, R. (2018). Is Peru Prepared for Large-Scale Sustainable Rural Electrification? Sustainability, 10(5), 1683. DOI: https://doi.org/10.3390/su10051683

Gaiddon, B. (2009). Photovoltaics in the Urban Environment. En Photovoltaics in the Urban Environment. earthscan. DOI: https://doi.org/10.4324/9781849770149

García, L. F. (2016). Evaluación de factibilidad de implementar generación distribuida fotovoltaica que permita aumentar la cobertura y confiabilidad de energía en sectores rurales del operador de red [Universidad Nacional de Colombia]. Recuperado de https://repositorio.unal.edu.co/bitstream/handle/unal/57904/2949944507.2016.pdf?sequence=1

Hachem, C. (2015). Design of a base case mixed-use community and its energy performance. Energy Procedia, 78, 663-668. DOI: https://doi.org/10.1016/j.egypro.2015.11.056

Hermida, María A; Hermida, Carla; Cabrera, Natasha; Calle, C. (2015). La densidad urbana como variable de análisis de la ciudad. El caso de Cuenca, Ecuador. Eure, 41(124), 25-44. DOI: https://doi.org/10.4067/s0250-71612015000400002

Inarquia. (2019). Web de Eficiencia Energética, Energías Renovables y Arquitectura / Inarquia. Inarquia. Recuperado de https://inarquia.es/

Izquierdo, I. F., Pacheco, M. G., González, L. G., \& Zalamea, E. F. (2019). Simulación fotovoltaica considerando parámetros de integración en edificaciones. Ingenius. Revista de Ciencia y Tecnología. DOI: https://doi.org/10.17163/ings.n21.2019.02

Kaan, H., \& Reijenga, T. (2004). Photovoltaics in an architectural context. Progress in Photovoltaics: Research and Applications, 12(6), 395-408. DOI: https://doi.org/10.1002/pip.554

Lin, Z., \& Qi, J. (2017). Hydro-dam - A nature-based solution or an ecological problem: The fate of the Tonlé Sap Lake. Environmental Research, 158(May), 24-32. DOI: https://doi.org/10.1016/j.envres.2017.05.016

Martínez, A. (2016). Energía solar fotovoltaica integrada en la edificación: modelizado y análisis del efecto del sombreado en la captación de irradiación. [Universidad de la Rioja]. Recuperado de https://dialnet.unirioja.es/descarga/tesis/50242.pdf

MEER. (2017). Balance Energético Nacional 2017. Recuperado de http://biblioteca.olade.org/opactmpl/Documentos/cg00701.pdf

Muñoz, J., Rojas, M., \& Barreto, C. (2018). Incentivo a la generación distribuida en el Ecuador. En Ingenius. Revista de Ciencia y Tecnología (pp. 60-68). scielo. Recuperado de https://www.researchgate.net/publication/322213432 Incentivo a la generacion distribuida en el E cuador

Ordenes, M., Marinoski, D. L., Braun, P., \& Rüther, R. (2007). The impact of building-integrated photovoltaics on the energy demand of multi-family dwellings in Brazil. Energy and Buildings, 39(6), 629-642. DOI: https://doi.org/10.1016/j.enbuild.2006.10.006

Poggi, F., Firmino, A., \& Amado, M. (2018). Planning renewable energy in rural areas: Impacts on occupation and land use. Energy, 155, 630-640. DOI: https://doi.org/10.1016/j.energy.2018.05.009

ACE, 15 (4.5) CC BY-ND 3.0 ES | UPC Barcelona, España | Capacidad e integración fotovoltaica en edificios 23 multifamiliares de mediana altura en la región ecuatorial andina. DOI: http://dx.doi.org/10.5821/ace.15.45.9307 
Ponce-Jara, M. A., Castro, M., Pelaez-Samaniego, M. R., Espinoza-Abad, J. L., \& Ruiz, E. (2018). Electricity sector in Ecuador: An overview of the 2007-2017 decade. Energy Policy, 113, 513-522. DOI: https://doi.org/10.1016/j.enpol.2017.11.036

ProViento S.A. Energías Renovables Ecuador. (2019). PROVIENTO - Paneles Solares. ProViento S.A. Energías Renovables Ecuador. Recuperado de https://proviento.com.ec/10-paneles-solares

QCELLS. (2015). Q.ANTUM SOLAR MODULE Q.plus-g4.3 280-290. Recuperado de https://www.qcells.com/dam/jcr:6249cd06-ade0-4872-b96b-

3ffefe1e49d9/Hanwha Q CELLS Data sheet QPLUS-G4.3 280-290 2017-12 Rev02 EN.pdf

Richardson, L. (2019). Thin Film Solar Panels: Do They Make Sense? / EnergySage. EnergySage. Recuperado de https://news.energysage.com/thin-film-solar-panels-make-sense/

Rosas-Flores, J. A., Zenón-Olvera, E., \& Gálvez, D. M. (2019). Potential energy saving in urban and rural households of Mexico with solar photovoltaic systems using geographical information system. Renewable and Sustainable Energy Reviews, 116, 109-412. DOI: https://doi.org/10.1016/j.rser.2019.109412

Rüther, R., \& Braun, P. (2009). Energetic contribution potential of building-integrated photovoltaics on airports in warm climates. Solar Energy, 83(10), 1923-1931. DOI: https://doi.org/10.1016/i.solener.2009.07.014

Sánchez, N., Vidal, R., \& Pastor, M. C. (2018). Aesthetic impact of solar energy systems. Renewable and Sustainable Energy Reviews, 98, 227-238. DOI: https://doi.org/https://doi.org/10.1016/j.rser.2018.09.021

Shukla, A. K., Sudhakar, K., \& Baredar, P. (2016). Simulation and performance analysis of 110 kWp gridconnected photovoltaic system for residential building in India: A comparative analysis of various PV technology. Energy Reports, 2, 82-88. DOI: https://doi.org/10.1016/j.egyr.2016.04.001

Solar Review. (2020, mayo 22). Latest Tier-1 Solar Panels List 2020 (Q1, Q2 update). Solar Review. Recuperado de https://review.solar/latest-tier-1-solar-panels-list-2020/

Solargis. (2017). Global Solar Atlas, World. Global Solar Atlas. Recuperado de https://globalsolaratlas.info/map?c=11.609193,8.261719,3\%0Ahttps://globalsolaratlas.info/?m=sg:ghi\% oAhttps://olc.worldbank.org/content/global-solaratlas\%OAhttps://globalsolaratlas.info/downloads/world

Sunfields - Europe. (2019). Panel SunPower® Performance para instalaciones de uso residencial. Recuperado de https://www.sfe-solar.com/wp-content/uploads/2019/12/P19_320_BLK_RES_ES-DIC2019.pdf

SunPower. (2020). Solar Panels / SunPower. Recuperado de https://us.sunpower.com/products/solarpanels

Tama, A. (2013). Cocina De Inducción Versus Cocina a Gas ( GLP ). Revista Técnica del Colegio Regional de Ingenieros Eléctricos y Electrónicos del Litora, 8-14. Recuperado de http://www.dspace.espol.edu.ec/handle/123456789/25742

United Nations. (2015). World Population Prospects - Population Division - United Nations. The International Journal of Logistics Management, 9, 1-13. DOI: https://doi.org/10.1108/09574099810805708 
Urgilés, B., Chávez, C., \& Espinoza, H. (2015). Hidroelectricidad en Ecuador. En M. Peláez \& J. Espinoza (Eds.), Energías renovables en el Ecuador. Situación actual, tendencias y perspectivas (Primera ed, pp. 116-157). Gráficas Hernández. Recuperado de https://www.researchgate.net/publication/291356953 Energia solar en el Ecuador

Usón Guardiola, E., Fumado Alsina, J. L., \& Vives Rego, J. (2014). Los edificios de consumo energético casi nulo: Propuesta para el centro de tratamiento de residuos sólidos de Mercabarna. Architecture, City and Environment, 9(25), 13-42. DOI: https://doi.org/10.5821/ace.9.25.3620

Vicente Ramón, M. G. (2017). Desarrollo de un sistema de informacion energético en el consumo de combustibles derivados de hidrocarburos en la jurisdicción de la regional de control de hidrocarburos y combustibles - Azuay [Universidad de Cuenca]. Recuperado de http://dspace.ucuenca.edu.ec/handle/123456789/27778

Villalta, L. J., \& Buñay, S. I. (2016). Evaluación energética-económica en el uso de calentadores de agua en el cantón Paute [Universidad de Cuenca]. Recuperado de http://dspace.ucuenca.edu.ec/handle/123456789/25837

Voss, K., \& Musall, E. (2012). Net zero energy buildings: International projects of carbon neutrality in buildings (C. Hellsterm, K. Ahrend, \& J. Rackwitz (eds.); 2013. ${ }^{a}$, New ed., p. 192). DETAIL Green Books. Recuperado

de

https://books.google.com.ec/books?hl=es\&lr=\&id=afrTAAAAQBAJ\&oi=fnd\&pg=PR4\&dq=Net+zero+ene rgy+buildings:+International+projects+of+carbon+neutrality+in+buildings\&ots=uV719vbMPr\&sig=5QP MH4obP1CzxpM9hGOnKkzOmww\#v=onepage\&q=Net\%20zero\%20energy\%20buildings\%3A\%20Intern\& $\underline{f=f a l s e}$

Zalamea, E., \& Quesada, F. (2017). Criterios de integración de energía solar activa en arquitectura. Potencial tecnológico y consideraciones proyectuales. Revista de Arquitectura, 19(1), 65-79. DOI: https://doi.org/10.14718/revarq.2017.19.1.1018

Zhang, H., Jarić, I., Roberts, D. L., He, Y., Du, H., Wu, J., Wang, C., \& Wei, Q. (2020). Extinction of one of the world's largest freshwater fishes: Lessons for conserving the endangered Yangtze fauna. Science of the Total Environment, 710(8), 1-7. DOI: https://doi.org/10.1016/j.scitotenv.2019.136242 